\title{
The Wide-field Infrared Survey Explorer (WISE): Mission Description and Initial On-orbit Performance
}

\author{
Edward L. Wright ${ }^{1}$, Peter R. M. Eisenhardt ${ }^{2}$, Amy Mainzer ${ }^{2}$, Michael E. Ressler ${ }^{2}$, Roc M. \\ Cutri $^{3}$, Thomas Jarrett ${ }^{3}$, J. Davy Kirkpatrick ${ }^{3}$, Deborah Padgett ${ }^{3}$, Robert S. McMillan ${ }^{4}$, \\ Michael Skrutskie $^{5}$, S. A. Stanford ${ }^{6,7}$, Martin Cohen ${ }^{8}$, Russell G. Walker ${ }^{8}$, John C. \\ Mather ${ }^{9}$, David Leisawitz ${ }^{9}$, Thomas N. Gautier III ${ }^{2}$, Ian McLean ${ }^{1}$, Dominic Benford ${ }^{9}$, \\ Carol J. Lonsdale ${ }^{10}$, Andrew Blain ${ }^{13}$, Bryan Mendez ${ }^{16}$, William R. Irace ${ }^{2}$, Valerie Duval ${ }^{2}$, \\ Fengchuan $\mathrm{Liu}^{2}$, Don Royer ${ }^{2}$, Ingolf Heinrichsen ${ }^{2}$, Joan Howard ${ }^{11}$, Mark Shannon ${ }^{11}$, \\ Martha Kendall ${ }^{11}$, Amy L. Walsh ${ }^{11}$, Mark Larsen ${ }^{12}$, Joel G. Cardon ${ }^{12}$, Scott Schick ${ }^{15}$, Mark \\ Schwalm ${ }^{17}$, Mohamed Abid ${ }^{2}$, Beth Fabinsky ${ }^{2}$, Larry Naes ${ }^{14}$, Chao-Wei Tsai ${ }^{3}$ \\ wright@astro.ucla.edu
}

\begin{abstract}
The all sky surveys done by the Palomar Observatory Schmidt, the European Southern Observatory Schmidt, and the United Kingdom Schmidt, the InfraRed Astronomical Satellite and the 2 Micron All Sky Survey have proven to be extremely useful tools for astronomy with value that lasts for decades. The Wide-field Infrared Survey Explorer is mapping the whole sky following its launch on 14 December 2009. WISE began surveying the sky on 14 Jan 2010 and completed its first full coverage of the sky on July 17 . The survey will continue to cover the sky a second time until the cryogen is exhausted (anticipated in November 2010). WISE is achieving 5 sigma point source sensitivities better than $0.08,0.11,1$ and 6 mJy in unconfused regions on the ecliptic in bands centered at wavelengths of 3.4, 4.6, $12 \& 22 \mu \mathrm{m}$. Sensitivity improves toward the ecliptic poles due to denser coverage and lower zodiacal background. The angular resolution is $6.1^{\prime \prime}, 6.4^{\prime \prime}, 6.5^{\prime \prime} \& 12.0^{\prime \prime}$ at $3.4,4.6,12 \& 22 \mu \mathrm{m}$, and the astrometric precision for high SNR sources is better than $0.15^{\prime \prime}$.
\end{abstract}

${ }^{1}$ UCLA Astronomy, PO Box 951547, Los Angeles CA 90095-1547

${ }^{2}$ Jet Propulsion Laboratory, 4800 Oak Grove Drive, Pasadena, CA 91109

${ }^{3}$ Infrared Processing and Analysis Center, California Institute of Technology, Pasadena CA 91125

${ }^{4}$ University of Arizona, 1629 E. University Blvd., Tucson, AZ 85721, USA

${ }^{5}$ Department of Astronomy, University of Virginia, Charlottesville VA 22903

${ }^{6}$ Physics Department, University of California, Davis, CA 95616

${ }^{7}$ Institute of Geophysics and Planetary Physics, LLNL, Livermore, CA, 94551

${ }^{8}$ Monterey Institute for Research in Astronomy, 200 8th Street, Marina CA 93933

${ }^{9}$ NASA Goddard Space Flight Center, Greenbelt MD 20771

${ }^{10}$ National Radio Astronomy Observatory, Char-

\section{Introduction}

The Wide-field Infrared Survey Explorer (WISE) will complete a mid-infrared survey of the entire

\footnotetext{
lottesville VA 22903

${ }^{11}$ Ball Aerospace \& Technologies Corporation, 1600 Commerce Street, Boulder, CO 80301

${ }^{12}$ Space Dynamics Laboratory, 1695 North Research Park Way, North Logan, UT 84341

${ }^{13}$ California Institute of Technology, Pasadena CA 91125

${ }^{14}$ Lockheed Martin Advanced Technology Center (retired), Palo Alto, CA

${ }^{15}$ Practical Technology Solutions, Inc., P.O Box 6336, North Logan, Utah 84341

${ }^{16}$ Space Sciences Laboratory, University of California, Berkeley CA 94720

${ }^{17}$ L-3 Communications SSG-Tinsley, Wilmington, MA 01887
} 
sky by mid-July 2010 with much higher sensitivity than previous infrared survey missions. The most comparable previous mission, the InfraRed Astronomical Satellite (IRAS; Neugebauer et al. 1984; Beichman et al. 1988) was launched in 1983 and mapped the whole sky in 4 bands with 62 individually wired detectors. The AKARI mission (Murakami et al. 2007) used an ingeneous technique to survey the mid-IR sky at $9 \& 18 \mu \mathrm{m}$ with sensitivities of $50 \& 100 \mathrm{mJy}$ (Ishihara et al. 2010) with better angular resolution than IRAS. WISE is mapping the whole sky in 4 infrared bands W1..W4 centered at 3.4, 4.6, $12 \& 22 \mu \mathrm{m}$ using a $40 \mathrm{~cm}$ telescope feeding arrays with a total of 4 million pixels. The increased number of detectors leads to a much higher sensitivity: WISE is achieving a sensitivity more than one hundred times better than IRAS in the $12 \mu \mathrm{m}$ band. While IRAS had two far-infrared bands at 60 and 100 $\mu \mathrm{m}$, WISE has two mid-infrared bands at $3.4 \&$ $4.6 \mu \mathrm{m}$. In these bands the only all-sky survey that has been done is from the Diffuse InfraRed Background Experiment (DIRBE) on the COBE satellite, and DIRBE used only a single pixel per band observing a $0.7^{\circ}$ beam. A point source catalog was constructed by Smith et al. (2004) from the DIRBE data with flux limits of $60 \& 50 \mathrm{Jy}$ at $3.5 \& 4.9 \mu \mathrm{m}$, and WISE should reach flux limits that are $5 \times 10^{4}$ times lower in these bands.

\section{Implementation}

WISE is a MIDEX (medium class Explorer) mission funded by NASA. The project is managed and operated by the Jet Propulsion Laboratory (JPL) for the PI, Edward L. Wright. Major components of the project were built by contractors, with the Space Dynamics Laboratory (SDL) of Utah State University building the instrument and Ball Aerospace \& Technologies Corporation building the spacecraft. See Liu et al. (2008) for more details about the development of the WISE flight system. The data processing and analysis is being done by the Infrared Processing and Analysis Center (IPAC). Education and Public Outreach activities for WISE are led by the UC Berkeley Space Sciences Lab (Méndez 2008).

The WISE flight system is $285 \mathrm{~cm}$ tall, $200 \mathrm{~cm}$ wide and $173 \mathrm{~cm}$ deep. It has a mass of $661 \mathrm{~kg}$. It uses $301 \mathrm{~W}$ of power, while the solar panels can

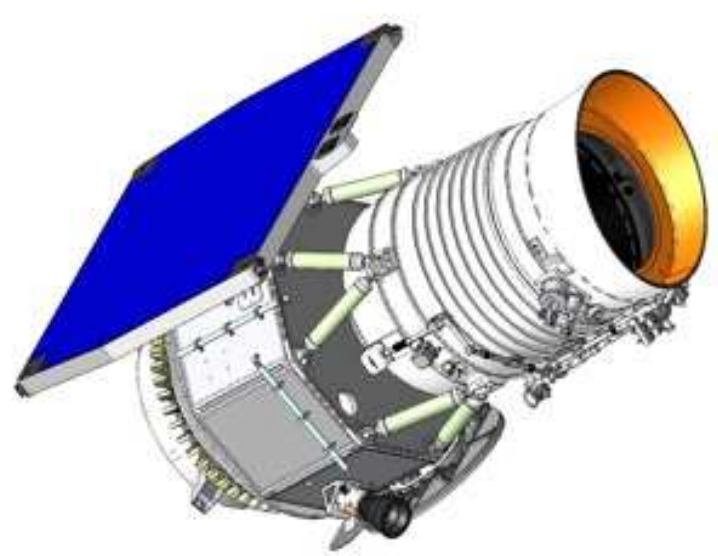

Fig. 1.- Diagram showing the WISE flight system in survey configuration with cover off. The spacecraft bus to the left of the bipod supports was provided by BATC, and the cryogenic instrument to the right of the bipods was provided by SDL.

provide over $500 \mathrm{~W}$. The $40 \mathrm{~cm}$ diameter telescope sits inside a solid-hydrogen cooled cryostat. The cryostat plus telescope and camera have a mass of $347 \mathrm{~kg}$, and the solid hydrogen has a mass of 15.7 $\mathrm{kg}$ at launch. Figure 1 shows the flight system in operational configuration.

\subsection{Mission Design}

It is easiest to observe sources when the Sun is not in the same part of the sky, since maximizing the elongation from the Sun makes it much easier to keep optics cool, and to avoid scattered light from the Sun. But to scan the entire sky one must observe the ecliptic poles, which are always $90^{\circ}$ from the Sun. Thus WISE adopted a basic strategy of scanning great circles with a center located at the Sun, which keeps the solar elongation at $90^{\circ}$. These circles scan from the North Ecliptic Pole (NEP) to the South Ecliptic Pole (SEP), crossing the ecliptic at longitudes $\pm 90^{\circ}$ from the Sun. The entire sky is covered by this scan pattern in half a year as the Earth-Sun line turns by $180^{\circ}$.

Confusion noise would prevent detection of key WISE science targets unless the beam size were less than 50 square arc-seconds, and since there 


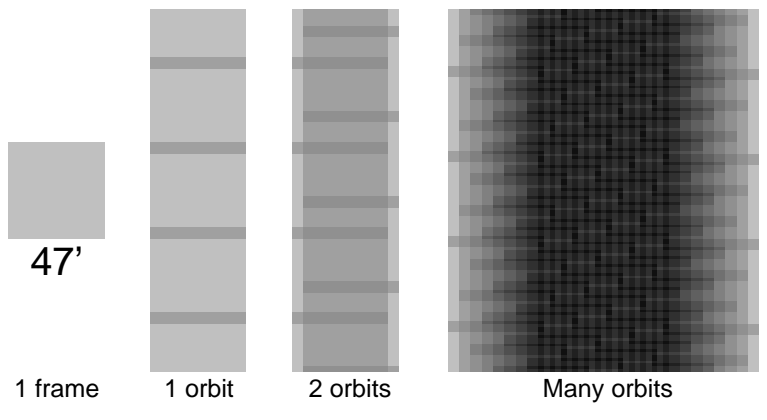

Fig. 2.- Coverage by WISE in one frame (every 11 seconds), in one orbit, in two orbits, and in many orbits. The gray levels show the depth of coverage with the darker areas having more coverage..
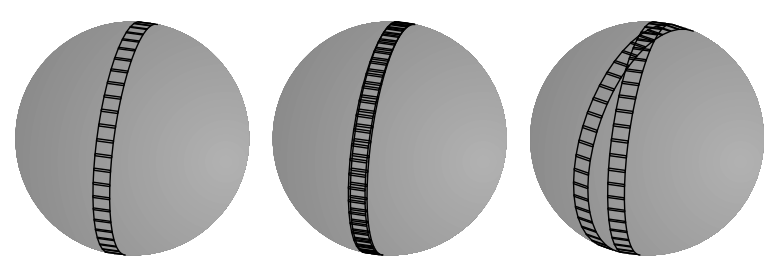

Fig. 3.- Cartoon showing WISE coverage on the sphere for 1 orbit, for 2 consecutive orbits, and for 2 orbits separated by 20 days, illustrating the highly redundant coverage at the ecliptic poles.

are $5 \times 10^{11}$ square arc-seconds in the sky, WISE clearly needs to transmit large quantities of data to the ground. This led to choosing a low Earth orbit to minimize the transmission loss. The actual WISE orbit started with a mean radius of 6909 $\mathrm{km}$ in mid-December 2009, and the radius had decreased by $0.8 \mathrm{~km}$ due to atmospheric drag by mid-June 2010. In a polar orbit the average geoid height is $\left(R_{e q}+R_{p}\right) / 2=6368 \mathrm{~km}$, so the average altitude above the geoid is $540 \mathrm{~km}$. This altitude was chosen to reduce the exposure to trapped radiation belts in the South Atlantic Anomaly (SAA). Since looking at the Earth would both blind the detector arrays and overwhelm the cryogenic system, a Sun synchronous orbit was chosen, with an inclination $\left(97.5^{\circ}\right)$ chosen to give a precession rate of $360^{\circ}$ in one year. The right ascension of the ascending node was chosen so that the local time at the equator crossings is either $6 \mathrm{AM}$ or $6 \mathrm{PM}$. For the actual WISE launch at 14:09:33 UT on 14 Dec 2009 the ascending node is at $6 \mathrm{PM}$ local time. Since the end of the orbit normal close to the Sun

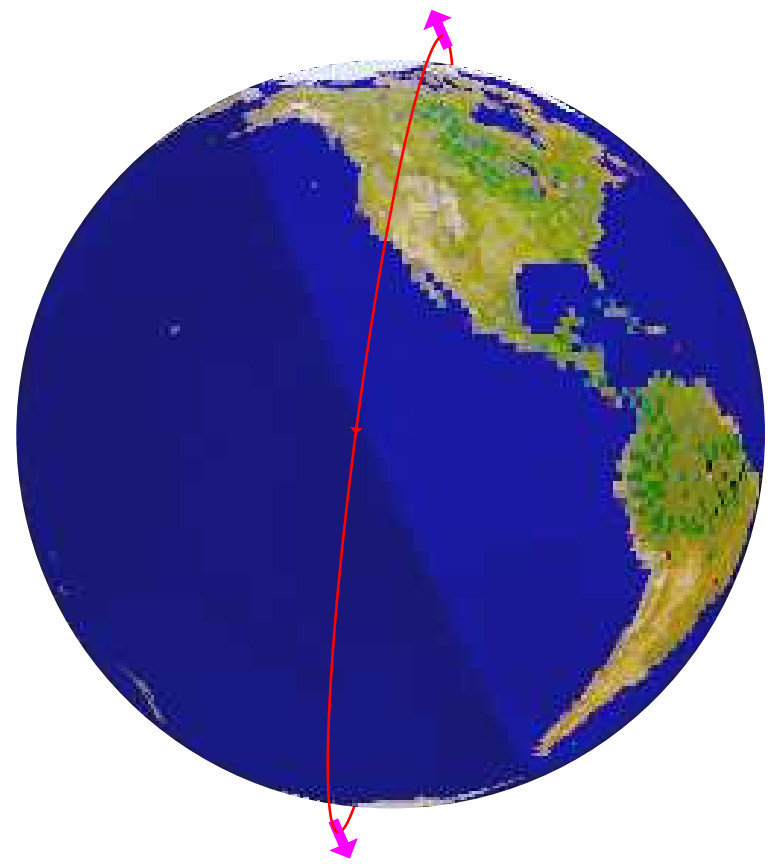

Fig. 4.- WISE pointing and orbit during the June solstice. Note that WISE points perpendicular to the Earth-Sun line and not toward the zenith.

is at $-7.5^{\circ}$ declination for dawn launch from the Western Test Range (at Vandenberg AFB, CA), during June the angle between Sun and the orbit normal can be as large as $31^{\circ}$, and eclipses occur over the South pole. COBE was in a $99^{\circ}$ inclination orbit at $900 \mathrm{~km}$ altitude with a June-centered eclipse season. The alternative of a $6 \mathrm{PM}$ launch from the WTR gives an eclipse season centered on the December solstice, as was the case for IRAS.

By synchronizing the scans and the orbit so the telescope is pointed at the NEP when the satellite is closest to the North pole of the Earth, one achieves a scan pattern that covers the entire sky, with the line of sight (LOS) never closer to the Sun than $90^{\circ}$ and never further than $31^{\circ}$ from the zenith.

WISE has adopted the 2MASS strategy of moving the telescope at a constant inertial rate, and using a scan mirror to freeze the sky on the arrays for the integration time. The field of view (FOV) of WISE is $47^{\prime}$, and by choosing to have a small $(10 \%)$ overlap between frames, the cadence between frames is set at 11.002 seconds per frame. 


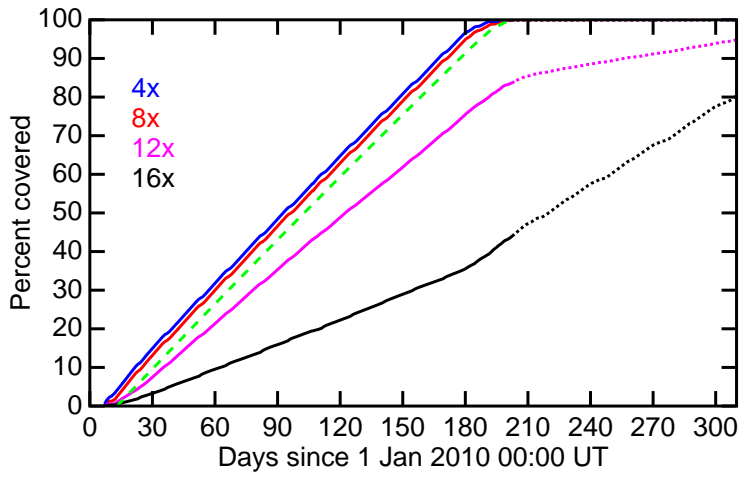

Fig. 5.- WISE survey coverage vs. date. As of 15 July 2010 more than $99 \%$ of the sky had been covered to depth of 8 frames or more. The fraction of the sky covered to a depth of 4,12 or 16 frames is also shown. The dotted portions of the curves show the anticipated coverage until the expected exhaustion of the cryogen. The vertical dashed line shows when the $22 \mu \mathrm{m}$ channel saturated after the actual exhaustion of the secondary tank. The small slowdowns in the survey progress occur when the Moon crosses the scan path. The dashed line shows a simple prediction of the sky coverage based only on the longitude of the Sun, starting on 14 Jan 2010.

For WISE 8.8 seconds of this is spent integrating and it takes about 1.06 seconds to read out the arrays. The LOS then jumps forward $42^{\prime}$ in 1.1 seconds. There are about 15 orbits per day, so the scan circle advances by about $4^{\prime}$ per orbit, giving about 12 orbits where the FOV hits a given source. Figure 2 shows how the redundancy builds up, while Figure 3 shows scans on the celestial sphere. Figure 4 shows the the relationship between the WISE orbit, the Earth-Sun line, and WISE line-of-sight during the eclipse season.

The Moon crosses the scan circle twice a month, and this would leave gaps in the sky coverage because the Moon is bright enough to cause stray light problems when it is less than $15^{\circ}$ from the line of sight. Thus WISE uses a modified scan pattern where the scan circle gets slightly ahead before the Moon interferes, and then drops slightly behind to recover the region the Moon blotted out. The Moon moves $13^{\circ}$ per day in ecliptic longitude, so with a $15^{\circ}$ exclusion zone WISE needs to be $1.2^{\circ}$ ahead just before the Moon crosses the scan

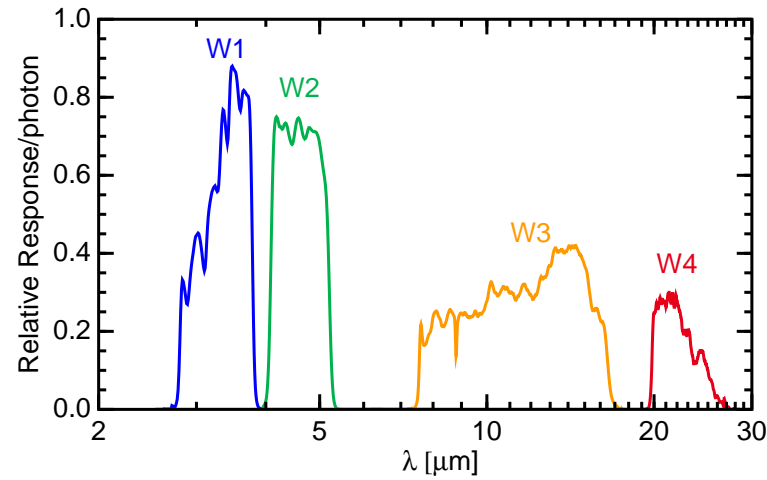

Fig. 6.- The weighted mean WISE relative spectral response functions in electrons per photon.

circle, and then drop back to $1.2^{\circ}$ behind. In addition, the SAA affects part of 4 orbits per day, and to mitigate reductions in the sky coverage due to the SAA, a small toggle of $\pm 0.2 \mathrm{deg}$ is added to the longitude of the scan circle center, with the sign changing with a two orbit period. Finally, the scan circle central longitude is biased by $+5^{\circ}$ on the side of the scan where the Sun is approaching the scan, which is the 6 PM side of the orbit. This means that the scans are actually $90^{\circ}$ and $95^{\circ}$ from the Sun when crossing the ecliptic, allowing for a 5 day recovery period after a satellite anomaly without causing a gap in sky coverage.

The WISE mission design provides at least 8 frames on over $99 \%$ of the sky in a 6 month survey interval after allowing for data lost to the Moon and the SAA. Heinrichsen \& Wright (2006) describe the mission operations system in more detail. Figure 5 shows the survey progress vs. date. In mid-July 2010 WISE completed its first pass over the sky, but will continue to survey until its cryogen runs out.

Duval et al. (2004) and Mainzer et al. (2005) describe the WISE hardware design.

\subsection{Instrument}

The WISE telescope, imager, dichroic beamsplitter, detectors, and cryostat were built by the Space Dynamics Laboratory of Utah State University with major subcontracts from Lockheed Martin Advanced Technology Center, L3 Communications SSG-Tinsley, and DRS Technologies. Larsen \& Schick (2005) describe the WISE science payload in more detail. The instrument 


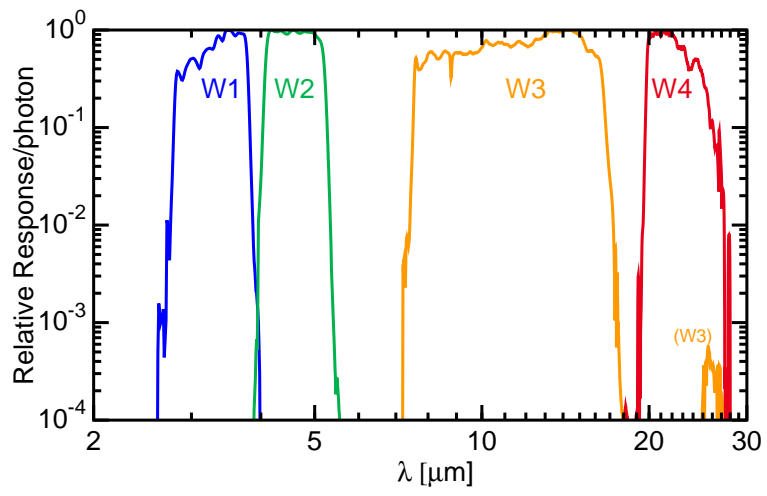

Fig. 7.- The weighted mean WISE relative spectral response functions after normalizing to a peak value of unity, on a logarithmic scale.

was tested, then passed a vibration test, and was tested again, before being delivered to Ball Aerospace in May 2009 for final integration with the spacecraft. The testing included a determination of the optimal focus, a measurement of the point spread function, and a determination of both the relative spectral response and the absolute sensitivity of the system.

The WISE short-wavelength channels employ 4.2 and $5.4 \mu \mathrm{m}$ cutoff $\mathrm{HgCdTe}$ arrays fabricated by Teledyne Imaging Sensors with $1024 \times 1024$ pixels each $18 \mu \mathrm{m}$ square. For the long-wavelength channels, the detectors are Si:As BIB arrays from DRS Sensors \& Targeting Systems with the same $1024 \times 1024$ pixel format and pitch. The first four and last four pixels in each row and column are used as non-illuminated reference pixels, so the effective size of the arrays is $1016 \times 1016$ pixels. The median pixel scale is $2.757^{\prime \prime} /$ pixel with a range of $\pm 0.6 \%$ among the different axes and arrays. The band 4 data is binned $2 \times 2$ on board, giving $5.5^{\prime \prime} /$ pixel. All four arrays image the same field of view simultaneously using 3 dichroic beam splitters.

All of the arrays use sampling up the ramp. The 11 sec cadence between frames is divided into 10 parts. A reset-read of the arrays occurs during the first $1.1 \mathrm{sec}$, followed by 8 read cycles each $1.1 \mathrm{sec}$ apart and a final reset cycle while the scan mirror flies back. For the W3 and W4 arrays the 9 reads are multiplied by weights of $-4,-3,-2,-1,0,1,2$, $3 \& 4$ and summed to give the slope of the ramp. The W1 and W2 arrays showed excess noise in the first sample so the weights used are $0,-7,-5,-3$, $-1,1,3,5 \& 7$.

The long-wavelength arrays show long lasting latent images in the flat-field which are removed by annealing these arrays twice per day. The annealing heaters are on for 90 seconds and heat the arrays from their $7 \mathrm{~K}$ normal operating temperature to $15 \mathrm{~K}$, which removes the latent images.

The spectral response of the system has been determined in three different ways: the whole system response was measured using a Fourier transform spectrometer (FTS), the system response was estimated using the product of component data (measured with an FTS), and the response was computed from component design calculations. Since the sharp edges in the response function cause ringing and negative values in the FTS spectrum, a "best" combined estimate of the relative spectral response was computed as follows: first a power law was fit to the ratio of the total system measured response to either the component prediction or the design prediction. The predictions were then adjusted to better match the measured transmission using these fits. The fits were weighted by the square of the response function times a $1 / \sigma^{2}$ statistical weight, so the correction is best in the filter passbands. After the adjustment the three methods gave consistent responses. Finally the best estimate for the spectral response was made using a weighted mean of the measured, predicted from components, and design values. In the weighted mean, zero or negative responses were given zero weight, the design and component predictions were given unit weight, and the measurements were given weights of $\mathrm{SNR}^{2}$. This weighted mean relative spectral response is shown in Figures 6] and 7 . The curves in the logarithmic plot have been normalized to a peak value of 1 . Note that the small red leak in the W3 transmission is based on data with a low SNR and may not actually exist.

WISE measures the signal $S$ given by

$$
S \propto \int R(\lambda) F_{\nu} d \ln \nu \propto \int R(\lambda) \lambda F_{\lambda} d \lambda
$$

since the relative spectral response $R$ is electrons per photon and $d n_{\gamma} \propto F_{\nu} d \nu /(h \nu) \propto F_{\nu} d \ln \nu$.

Specifying a central wavelength for broad bands like the WISE filters, especially the very wide W3 band, is always ambiguous. We have cho- 
TABLE 1

FLUX CORRECTIONS AND COLORS FOR POWERLAWS AND BLACKBODIES

\begin{tabular}{lrrrrrrr}
\hline \hline \multicolumn{1}{c}{$F_{\nu}$} & $f_{c}(W 1)$ & $f_{c}(W 2)$ & $f_{c}(W 3)$ & $f_{c}(W 4)$ & {$[\mathrm{W} 1-\mathrm{W} 2]$} & {$[\mathrm{W} 2-\mathrm{W} 3]$} & {$[\mathrm{W} 3-\mathrm{W} 4]$} \\
\hline \hline$\nu^{3}$ & 1.0283 & 1.0206 & 1.1344 & 1.0142 & -0.4040 & -0.9624 & -0.8684 \\
$\nu^{2}$ & 1.0084 & 1.0066 & 1.0088 & 1.0013 & -0.0538 & -0.0748 & -0.0519 \\
$\nu^{1}$ & 0.9961 & 0.9976 & 0.9393 & 0.9934 & 0.2939 & 0.8575 & 0.7200 \\
$\nu^{0}$ & 0.9907 & 0.9935 & 0.9169 & 0.9905 & 0.6393 & 1.8357 & 1.4458 \\
$\nu^{-1}$ & 0.9921 & 0.9943 & 0.9373 & 0.9926 & 0.9828 & 2.8586 & 2.1272 \\
$\nu^{-2}$ & 1.0000 & 1.0000 & 1.0000 & 1.0000 & 1.3246 & 3.9225 & 2.7680 \\
$\nu^{-3}$ & 1.0142 & 1.0107 & 1.1081 & 1.0130 & 1.6649 & 5.0223 & 3.3734 \\
$\nu^{-4}$ & 1.0347 & 1.0265 & 1.2687 & 1.0319 & 2.0041 & 6.1524 & 3.9495 \\
$B_{\nu}(100)$ & 17.2062 & 3.9096 & 2.6588 & 1.0032 & 10.6511 & 18.9307 & 4.6367 \\
$B_{\nu}(141)$ & 4.0882 & 1.9739 & 1.4002 & 0.9852 & 7.7894 & 13.0371 & 3.4496 \\
$B_{\nu}(200)$ & 2.0577 & 1.3448 & 1.0006 & 0.9833 & 5.4702 & 8.8172 & 2.4949 \\
$B_{\nu}(283)$ & 1.3917 & 1.1124 & 0.8791 & 0.9865 & 3.8329 & 5.8986 & 1.7552 \\
$B_{\nu}(400)$ & 1.1316 & 1.0229 & 0.8622 & 0.9903 & 2.6588 & 3.8930 & 1.2014 \\
$B_{\nu}(566)$ & 1.0263 & 0.9919 & 0.8833 & 0.9935 & 1.8069 & 2.5293 & 0.8041 \\
$B_{\nu}(800)$ & 0.9884 & 0.9853 & 0.9125 & 0.9958 & 1.1996 & 1.6282 & 0.5311 \\
$B_{\nu}(1131)$ & 0.9801 & 0.9877 & 0.9386 & 0.9975 & 0.7774 & 1.0421 & 0.3463 \\
$\mathrm{~K} 2 \mathrm{~V}$ & 1.0038 & 1.0512 & 1.0030 & 1.0013 & -0.0963 & 0.1225 & -0.0201 \\
$\mathrm{G} 2 \mathrm{~V}$ & 1.0049 & 1.0193 & 1.0024 & 1.0012 & -0.0268 & 0.0397 & -0.0217 \\
\hline \hline
\end{tabular}

sen to specify the isophotal wavelengths for our filters. The isophotal wavelength and magnitude zeropoint are defined by requiring that a constant $F_{\lambda}^{\circ}$ give the same signal as Vega in a WISE band, and that $F_{\lambda}^{\circ}=F_{\lambda}^{V e g a}\left(\lambda_{\text {iso }}\right)$. Tokunaga \& Vacca (2005) give a clear definition of the isophotal wavelength, and values for Mauna Kea Observatory filter set. Cohen et al. (1992) give the IR spectrum of Vega, based on a model from Kurucz (1991, private communication to Martin Cohen). It is important to remember that WISE saturates on Vega, so the magnitude zeropoint is actually based on fainter stars calibrated to the Vega system. Therefore the debris disk around Vega (Aumann et al. 1984) is relevant only if it has affected the calibration used to determine the magnitudes of the fainter standards. A convenient and accurate fit to the continuum of our Vega spectrum in the $2.5<\lambda<29 \mu \mathrm{m}$ range we use is given by

$$
\begin{aligned}
F_{\lambda}= & 1.0158 \times 10^{-16}\left(1-0.0083 \ln (\lambda / 8.891 \mu \mathrm{m})^{2}\right) \\
& \times B_{\lambda}(14454 \mathrm{~K})
\end{aligned}
$$

where $B_{\lambda}$ is the Planck function, which matches the continuum spectrum to an average absolute error of $0.045 \%$. The temperature in this ad hoc fit is much higher than the effective temperature of Vega and the solid angle is much lower than the real solid angle due to the increase with wavelength of the free-free and boundfree opacites. Absorption lines reduce the in-band fluxes by only $0.73,0.57,0.28 \& 0.14 \%$ in bands 1 through 4 . To avoid possible multiple solution we use the continuum spectrum when solving for $\lambda_{i s o}$. The isophotal wavelengths so defined are 3.3526, 4.6028, $11.5608 \& 22.0883 \mu \mathrm{m}$ for W1..4. The measured system transmissions were smaller than the expected values at long wavelengths, leading to an effective wavelength for WISE band 4 of $22 \mu \mathrm{m}$ instead of the expected $23 \mu \mathrm{m}$. Absolute measurements of Vega by MSX (Price et al. 2004) show that a 2.7\% upward offset from the model spectrum is needed at $21.3 \mu \mathrm{m}$ (Cohen 2009). We have applied this correction to the WISE $22 \mu \mathrm{m}$ band, giving magnitude zeropoints on the Vega system in the WISE passbands of $F_{\lambda}^{\circ}=8.180 \times 10^{-15}, 2.415 \times 10^{-15}, 6.515 \times$ $10^{-17} \& 5.090 \times 10^{-18} \mathrm{~W} / \mathrm{cm}^{2} / \mu \mathrm{m}$, which convert to $F_{\nu}^{\circ}=306.681,170.663,29.0448 \& 8.2839 \mathrm{Jy}$ 
in W1..W4 using $F_{\nu}^{\circ}=\left(\lambda_{i s o}^{2} / c\right) F_{\lambda}^{\circ}$. There is an overall systematic uncertainty of $\pm 1.5 \%$ from the Vega spectrum in these flux zeropoints.

We have also observed in-flight a discrepancy between red (typically sources with $F_{\nu} \propto \nu^{-2}$ ) and blue (stars with $F_{\nu} \propto \nu^{2}$ ) calibrators in W3 and $\mathrm{W} 4$, the 12 and $22 \mu \mathrm{m}$ bands. This amounts to about $-17 \%$ and $9 \%$ in the fluxes for $\mathrm{W} 3$ and $\mathrm{W} 4$, with the red sources appearing too bright in W4 and too faint in W3. The flux differences could be resolved by adjusting the effective wavelength of W3 and W4 3-5\% blueward and 2-3\% redward, respectively. This would change the zero magnitude $F_{\nu}^{\circ}$ by about $-8 \%$ in $\mathrm{W} 3$ and $+4 \%$ in $\mathrm{W} 4$. But the zeropoints and isophotal wavelengths reported here are based on the relative spectral responses derived from ground calibration without any such adjustment. The instrumental zeropoints that define the conversion from counts to magnitudes have been based on standard stars, which are the blue calibrators. Given the discrepancy between red and blue calibrators we estimate that the conversion from magnitudes to Janskys are currently uncertain by $\pm 10 \%$ in W3 and W4. Updated values will be provided in the Explanatory Supplement accompanying the preliminary data release.

This definition of the isophotal wavelength and flux zeropoint means that the color correction term for a source with a different spectrum than Vega vanishes by construction when $F_{\lambda}$ is a constant $\left(F_{\nu} \propto \nu^{-2}\right)$ and very nearly vanishes for Rayleigh-Jeans sources with $F_{\lambda} \propto \lambda^{-4}$ or $F_{\nu} \propto \nu^{2}$. These spectral energy distributions bracket the vast majority of WISE sources, so the the color corrections are generally small. But the extremely wide W3 filter does lead to color corrections as large as 0.1 magnitude for a constant $F_{\nu}$. Table 1 gives the flux correction factors in the WISE bands for several input spectra, and the WISE colors for these spectra. These factors multiply the signal $S$ given by a spectrum $F_{\nu}=F_{\nu}^{\circ}\left(\lambda_{\text {iso }} / \lambda\right)^{\beta}$. Thus a spectrum with $F_{\nu}=$ const $=29.0$ Jy gives a signal that is $f_{c}(W 3)=0.9169$ times the signal from Vega, so one would need a constant $F_{\nu}$ of $29.0 / 0.9169=31.7$ Jy to give zero magnitude.

Outer Solar system objects like Centaurs will have color temperatures close to $100 \mathrm{~K}$ and for a $100 \mathrm{~K}$ blackbody the flux correction factor $f_{c}(W 3)=2.6588$ is quite large. Thus a $100 \mathrm{~K}$ blackbody would need 29.0/2.66 = $10.9 \mathrm{Jy}$ at $11.56 \mu \mathrm{m}$ to give zero magnitude in $\mathrm{W} 3$.

The most common stars in the WISE catalog at high galactic latitude should be G-K dwarfs, so the flux correction factors and colors for a $\mathrm{K} 2 \mathrm{~V}$ and a G2V have been included in Table 1 using Kurucz (1993) spectra. Since the W2 band includes the fundamental $\mathrm{CO}$ bandhead, the flux correction factors give the ratio between the signal from the star to the signal from a constant $F_{\lambda}$ equal to the average over a log normal passband with a $9.1 \%$ FWHM centered on $\lambda_{i s o}$.

\subsubsection{Optics}

The optical design and assembly of the WISE telescope and camera was done by L-3 Communications SSG-Tinsley. The optics consist of an afocal $40 \mathrm{~cm}$ diameter telescope that produces a parallel beam of light that is fed into the scan mirror, which works in the parallel beam, and then into the all-reflecting camera. There are six mirrors including folding flats in the afocal telescope before the scan mirror, and six mirrors in the camera after the scan mirror. All of the mirrors are gold-coated giving a high infrared transmission. The design is described in more detail by Schwalm et al. (2005).

\subsubsection{Cryostat}

The cryostat (Naes et al. 2008) was built by the Lockheed Martin Advanced Technology Center. It uses solid hydrogen to cool the telescope to less than $12 \mathrm{~K}$, and the Si:As arrays to less than 7.5 $\mathrm{K}$. There are two tanks of solid hydrogen: a larger secondary tank that cools the telescope and optics, the short wavelength arrays, and shields around the smaller primary tank that just cools the $\mathrm{Si}: \mathrm{As}$ long wavelength arrays. It has a predicted onorbit lifetime of $10.75_{-0.5}^{+1}$ months. Schick \& Lloyd (2009) describe the cryostat support system and test results.

However, the in-flight performance deviated from the model predictions with the secondary tank running out of hydrogen on 5 August after 7.7 months in orbit. The primary tank continued to cool the long wavelength detectors, but the telescope warmed up to $46 \mathrm{~K}$ producing large backgrounds at $12 \& 22 \mu \mathrm{m}$. The $22 \mu \mathrm{m}$ channel stopped producing useful data after 8 August 
2010. The integration time on the $12 \mu \mathrm{m}$ channel was cut in half on 14 August, cut in half again on 20 August, and cut in half again on 23 August. Observations continued in the 3.4, $4.6 \& 12 \mu \mathrm{m}$ bands until the primary tank ran out of hydrogen on 29 Sep 2010.

\subsection{Spacecraft}

The WISE spacecraft was built by Ball Aerospace, with a design based on the RS300 series of single string spacecraft. The NextSat component of the Orbital Express mission was the first RS300 spacecraft to be launched, and it worked flawlessly. SWRI provided the spacecraft avionics.

WISE is a three-axis controlled spacecraft that is commanded to follow long scans at a constant inertial rate. The spacecraft provides attitude control of better than $75^{\prime \prime}$, jitter of less than $1.3^{\prime \prime}$, and drift rate variation of less than $0.2^{\prime \prime} / \sec$ over 9 seconds. Angular momentum is stored on board in 4 reaction wheels, and any buildup of excess angular momentum is dumped using magnetic torquer rods. Primary attitude information is provided by two Ball CT-633 star trackers. A fiber optic inertial measurement unit, Sun sensors and magnetometers are used during safe and emergency modes of the spacecraft.

A fixed solar panel provides over 500 watts of power. WISE is oriented so the solar panel is always pointing very nearly at the Sun. During eclipse, a 20 amp-hour lithium-ion battery provides flight system power. Science data and flight system telemetry are stored on a $96 \mathrm{~GB}$ flash memory card for later transmission to earth. The science data volume is about 50 Gbytes per day of uncompressed data, but lossless compression reduces this by roughly a factor of 2 . A fixed high gain antenna is used to transmit data to the Tracking and Data Relay Satellite System (TDRSS). The spacecraft body steers the antenna to TDRSS for downlink. Science operations have to be stopped during data transmission, so the data downlinks are scheduled while the satellite passes over the poles of the Earth, where the sky coverage is highly redundant.

\subsection{Mission Operations}

Except for looking up instead of down, WISE is very similar to an Earth observing satellite. WISE is in a Sun-synchronous low Earth orbit like the Landsats and many other Earth observers, and it uses the TDRSS to download a large volume of data. Thus WISE is being operated by the Earth Sciences Operations Center at JPL which also operates the JASON ocean topography mapping mission.

The preparation of a command load for WISE starts with a set of survey planning parameters, which then are used along with a predicted orbit to generate the survey plan for a week in the future. Times when a turn to TDRSS would be compatible with the WISE Sun and Earth pointing constraints are chosen, and a complete sequence of pointing commands covering half a week that combines observing with TDRSS downloads is created. Additional commands for momentum unloading and annealing the W3 and W4 detector arrays are inserted into the command sequence. This process is repeated twice a week to allow for the uncertainties in the predicted orbit caused by the fluctuating atmospheric drag due to solar activity. In the first half of 2010 the solar activity has been anomalously low, minimizing the errors in the predicted orbits.

\subsection{Data Processing \& Archiving}

WISE science data processing, archiving and distribution is performed by the Infrared Processing and Analysis Center (IPAC), California Institute of Technology. The processing software and operations system is based on algorithms, pipelines and architecture used for the 2MASS, Spitzer Space Telescope and GALEX projects.

Following each TDRSS downlink contact, science image data packets are sent to IPAC where they are uncompressed and assembled into raw FITS format images in the four WISE bands. The raw FITS image headers are populated with flight system engineering telemetry, navigation data and survey events information that is sent asynchronously from Mission Operations at JPL. The images from each downlink transfer are then passed to the WISE Scan/Frame pipeline that performs instrumental calibration (droop correction, linearization, bias subtraction, flat-fielding), source extraction, photometric and astrometric calibration and artifact identification on the individual exposure frames. The WISE Multiframe pipeline operates on collections of calibrated 
single-frame images that cover a region on the sky. The single-frame images are coadded by registering them onto a common pixel grid, matching local background levels, and optimally combining resampled pixel values using outlier rejection to suppress transient pixel events such as cosmic rays, noise excursions and fast moving objects (Masci \& Fowler 2009). Sources are detected and characterized on both the resulting combined images and individual frames, and spurious detections of image artifacts are identified and flagged. Both Scan/Frame and Multiframe processing are accompanied by detailed quality assessment to monitor data accuracy relative to WISEs science data requirements.

Source detection and characterization in the Scan/Frame and Multiframe pipelines exploit WISEs simultaneous four band measurements. Sources are detected by thresholding on a combined four-band, matched-filter image, similar to the "chi-square" detection method described by Szalay et al. (1999). Profile-fit photometry is performed on all bands simultaneously, using a maximum-likelihood fitting procedure that also operates on multiple, independent image frames in the Multiframe pipeline.

Astrometric calibration of single-exposure WISE images is performed by solving for the frame center positions and rotations using the in-situ measured position of 2MASS PSC astrometric reference stars. Band-to-band rotations and offsets, plate scales, and field distortion are determined by off-line analysis using the apparent positions of reference stars in thousands of image frames. Full WCS information derived from the astrometric solutions is included in the single-frame image headers. The single-frame astrometric solutions are propagated to the coadded images and extracted source lists in the Multiframe pipeline.

Processed WISE images and extracted source data and metadata will be archived and served to the community via the web-based and machinefriendly interfaces of the NASA/IPAC Infrared Science Archive (IRSA). WISE data products will be interoperable with other data centers and services through the IRSA infrastructure.

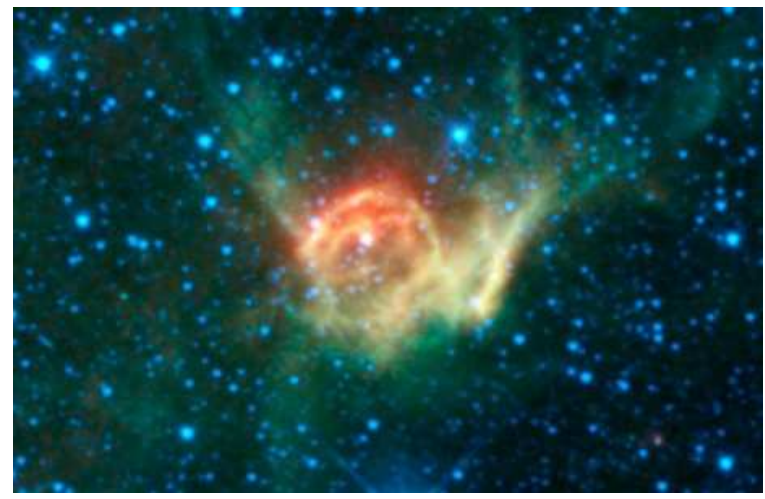

Fig. 8. - The Wolf-Rayet wind bubble NGC 2359 in the infrared using W1 as blue, W2 as cyan, W3 as green, and W4 as red. Image width is $0.4^{\circ}$, with North to the left and West up.

\section{On-orbit Performance}

WISE observes each patch of sky many times over a period of a day or more during its normal survey operation. These multiple frames can be combined into co-added multicolor mosaics such as Figure 8, which shows the the Wolf-Rayet wind bubble NGC 2359 (Schneps \& Wright 1980). The WISE project is posting an image of the week on its multimedia gallery which can be reached via http://wise.astro.ucla.edu

While the sensitivity of WISE is specified for 8 coverages, this is a worst case. Even on the ecliptic, where the coverage is the smallest, the most likely number of frames covering a given source is 11. The individual frames are analyzed to provide astrometric and photometric information. Analyzing the scatter among the values from individual frames gives the noises shown in Figure 9. The dashed line shows a 5:1 SNR in 8 frames scaled to the 11 frame case plotted, giving $\sigma(m)=0.185 \mathrm{mag}$ where $n$ is a noise term fitted to the data points. The curves show $\sigma(m)=0.01+[2.5 / \ln (10)] n / 10^{-0.4 m}$. The magnitudes where the curves cross the dashed line are $17.11,15.66,11.40 \& 7.97 \mathrm{mag}$. These give raw sensitivities of $44,93,800 \& 5500 \mu \mathrm{Jy}$ for $5 \sigma$ in 8 frames. There will be additional uncertainty due to source confusion which is not included in these noises. Allowing for confusion gives sensitivites of $0.08,0.11,1$ \& 6 mJy.

The astrometric accuracy of WISE can be es- 


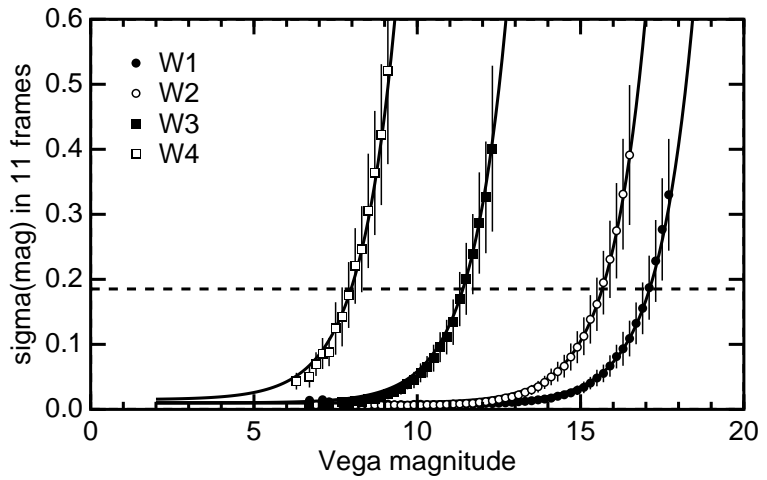

Fig. 9.- Standard deviation of WISE magnitudes derived from the repeatability on sources seen in 11 frames. The standard deviations have been binned into bins with width $0.2 \mathrm{mag}$, and the resulting histograms have been fit with a gaussian using outlier rejection. The points and errorbars show the derived means and standard deviations. The curves show a noise model fitted to the data. The dashed line shows an SNR of 5.86:1 which corresponds to $5: 1$ in 8 frames. From left to right, the open squares show $\mathrm{W} 4$, the filled squares show W3, the open circles show W2, and the filled circles show W1.

timated by looking at the differences between WISE and UCAC3 (Zacharias et al. 2010) positions, shown in Figure 10 for sources with SNR $>$ 20. At lower SNRs, an additional error of about FWHM/(2 SNR) must be added in quadrature. Since this additional error is $0.15^{\prime \prime}$ for a PSF FWHM of $6 \mathrm{sec}$ and $\mathrm{SNR}=20$, and the observed width of the distribution implies $\sigma=0.17^{\prime \prime}$ for SNR $>20$, WISE positions for high SNR sources should be better than $0.15^{\prime \prime}$ for $1 \sigma$ and 1 axis.

The image quality for WISE is good, as shown in Figure 11. These curves show the azimuthally averaged point spread function for WISE with FWHMs of $6.1^{\prime \prime}, 6.4^{\prime \prime}, 6.5^{\prime \prime} \& 12.0^{\prime \prime}$ in WISE bands 1 to 4 . In the geometric limit without diffraction the estimated FWHM is roughly 5.5".

The sensitivity of WISE to diffuse emission can be estimated from the point source sensitivity and the beam size. The point source flux sensitivity scales like the square root of the noise effective solid angle $\Omega_{e}$ (Wright 1985), also known as noise pixels. Thus the diffuse sensitivity for a source solid angle $\Omega$ is given by

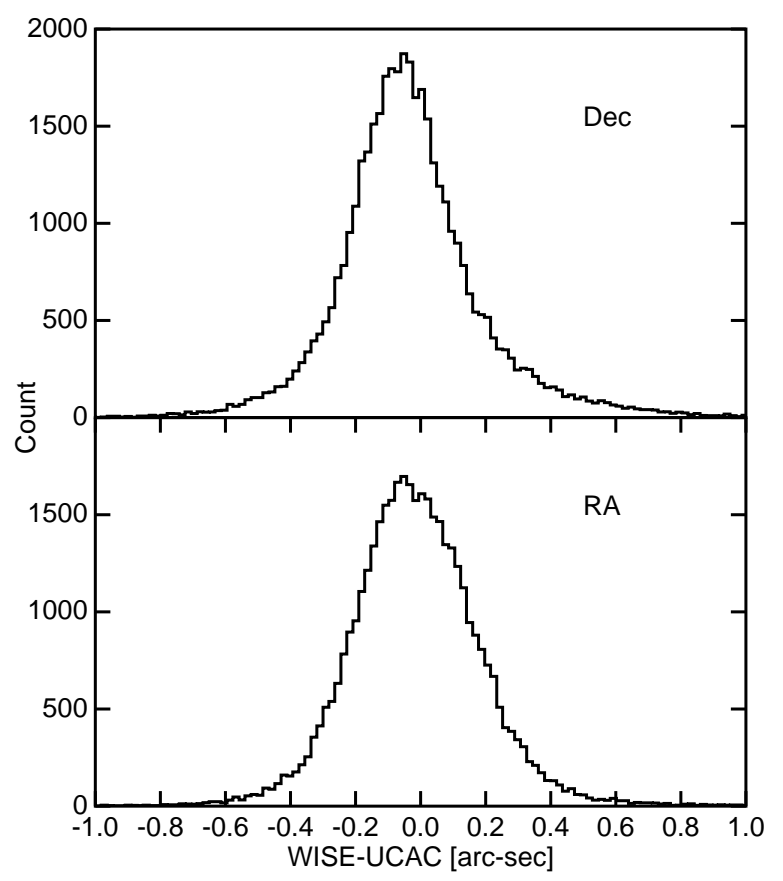

Fig. 10.- Histograms of the difference in right ascension and declination between WISE and UCAC positions for objects with SNR in W1 $>20$.

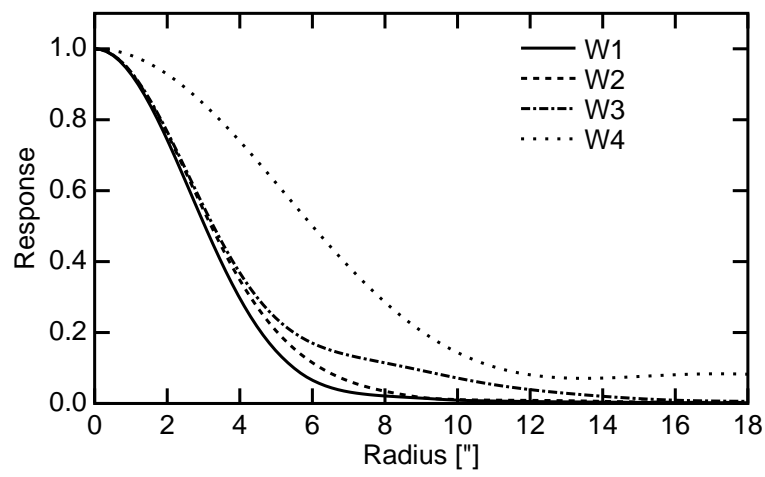

Fig. 11.- Azimuthally averaged point spread functions for WISE. 


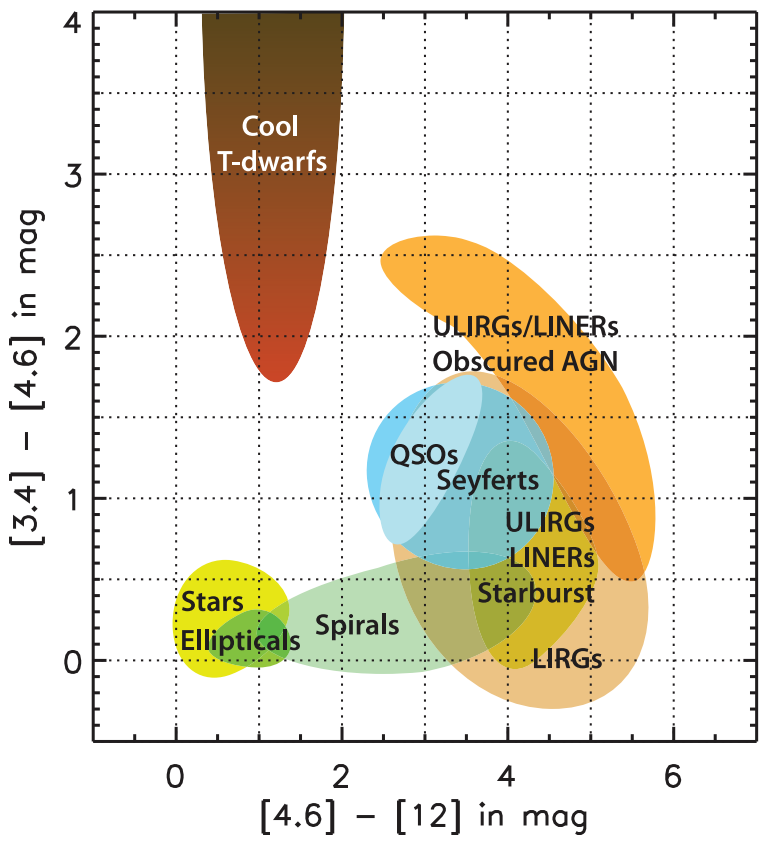

Fig. 12.- Color-color diagram showing the locations of interesting classes of objects. Stars and early type galaxies have colors near zero, while brown dwarfs are very red in W1-W2, spiral galaxies are red in W2-W3, and ULIRGS tend to be red in both colors.

$\sigma(I)=\sigma(F) / \sqrt{\Omega_{e} \Omega}$. The noise pixels for W1..W4 are $13.4,16.7,34.3\left(2.75^{\prime \prime}\right)$, and $25.9\left(5.5^{\prime \prime}\right)$. These give $\Omega_{e}=2.4,3.0,6.1 \& 18.4 \mathrm{nsr}$ in $\mathrm{W} 1 . . \mathrm{W} 4$. For a source size of $5^{\prime} \times 5^{\prime}$ or $\Omega=2.1 \mu \mathrm{sr}$, the $5 \sigma$ diffuse sensitivities from statistical noise alone are $0.6,1.2,7 \& 28 \mathrm{kJy} / \mathrm{sr}$ in W1..W4. The sensitivity to large scale structures will also be limited by errors in the flat field corrections and inaccuracies in matching the backgrounds from frame to frame.

The observed saturation levels for WISE are $0.3,0.5,0.7 \& 10 \mathrm{Jy}$ for W1..W4. Saturated pixel values are replaced with a flag indicating which of the 9 samples up the ramp was the first to saturate, so there is still a coarse indication of the brightness in saturated regions.

\section{Science Goals}

WISE has achieved $5 \sigma$ point source sensitivities better than $0.08,0.11,1 \& 6$ mJy at $3.4,4.6,12$ $\& 22 \mu \mathrm{m}$ in regions observed in 8 or more frames, which is expected to be more than $99 \%$ of the sky.

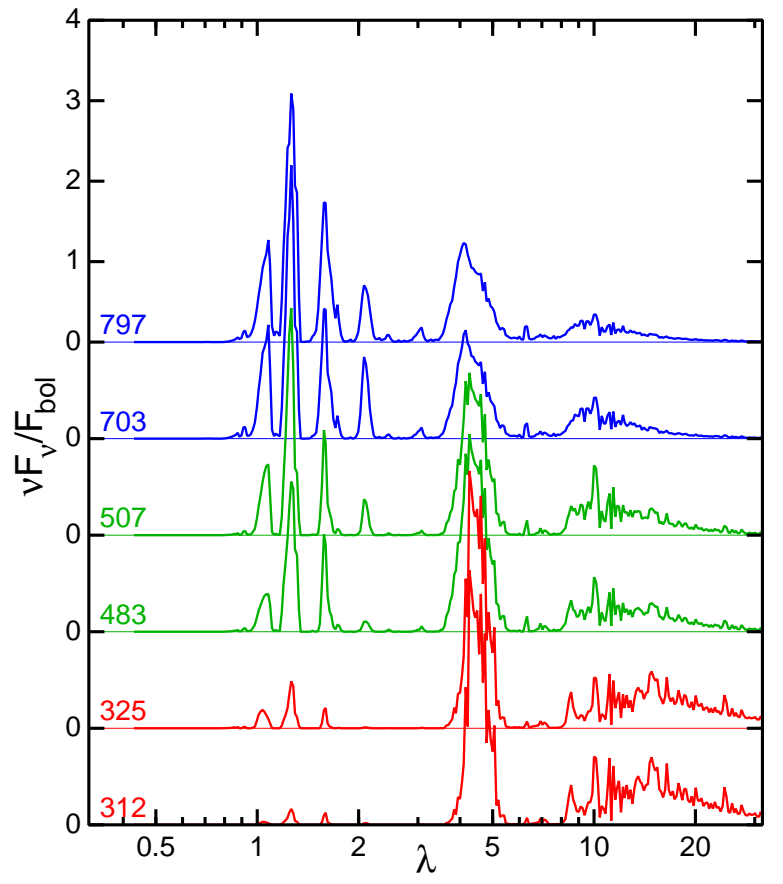

Fig. 13. - Brown dwarf spectra selected from Burrows et al. (2003) smoothed to 1\% resolution. Curves are labeled with the effective temperature from $797 \mathrm{~K}$ at the top to $312 \mathrm{~K}$ at the bottom.

These sensitivities correspond to Vega magnitudes of $16.5,15.5,11.2 \&$ 7.9. Thus WISE will go a magnitude deeper than the 2MASS $\mathrm{K}_{s}$ data in W1 for sources with spectra close to that of an A0 star, and even deeper for moderately red sources like K stars or galaxies with old stellar populations.

The fundamental objective of WISE is to provide a sensitive all-sky survey in the mid-infrared. This survey will probably provide its biggest payoff in ways that have not yet been imagined. But several fields can be identified where an all-sky survey will provide a guaranteed payoff.

Many classes of extragalactic objects will be quite red in the WISE colors, as will brown dwarf stars which are among the Sun's closest neighbors. Figure 12 shows the color-color diagram constructed from the W1..W3 bands, with the regions occupied by various types of objects illustrated.

\subsection{Brown Dwarf Stars}

Brown dwarf stars are very faint, since they are not massive enough to fuse hydrogen into he- 


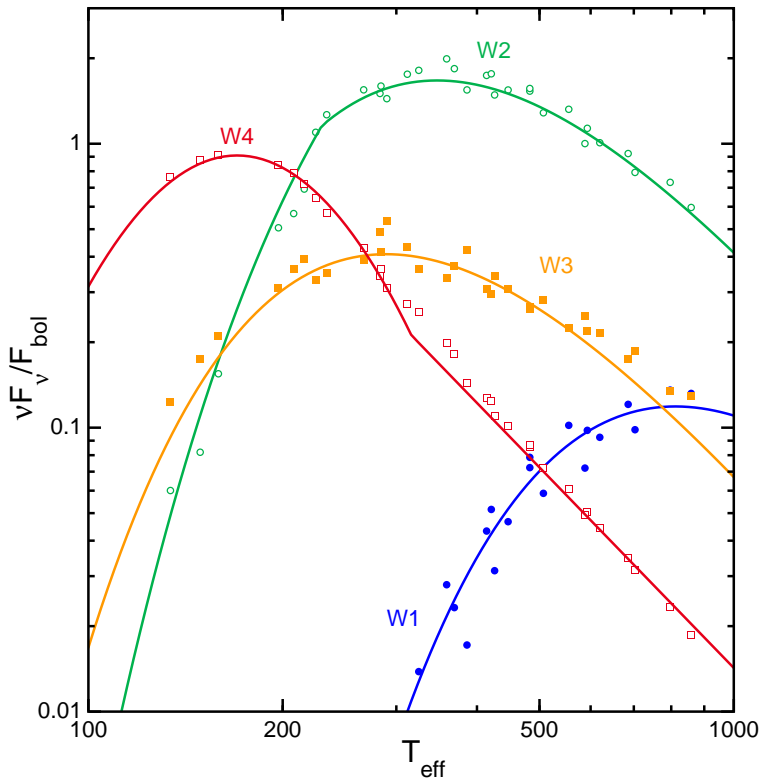

Fig. 14. - Flux in the WISE bands compared to the bolometric flux as a function of $T_{\text {eff }}$ for the models of Burrows et al. (2003). Simple functional fits are shown.

lium. As a result, they gradually fade and cool, and old brown dwarfs will be very cool and faint. Both Jupiter with $L=10^{-9} L_{\odot}$ and Gliese 229B with $L=10^{-5} L_{\odot}$ have very strong emission at $4.6 \mu \mathrm{m}$ due to a lack of methane absorption at this wavelength (Kirkpatrick 2005). Thus the 4.6 $\mu \mathrm{m}$ band of WISE is a powerful tool for finding cool brown dwarfs. Most of the brown dwarfs found prior to WISE have been discovered using the $z^{\prime}$ band of SDSS or the $J$ band of 2MASS or UKIDSS, but fairly high temperatures are required before there is substantial emission in either of these short wavelength bands. As a result the currently known sample of brown dwarfs is biased toward the hotter, and thus younger, objects. WISE is able to find 10 Gyr old brown dwarfs and thus should detect a high density of stars in the solar neighborhood. The expected number density of brown dwarfs is 1-2 times the density of ordinary stars (Reid et al. 1999; Chabrier 2002).

The expected number of brown dwarfs that WISE will see can be computed using models for the emitted spectra of brown dwarfs and the luminosity and effective temperature of brown dwarfs as a function of mass and age Burrows et al.
2003). These model spectra are plotted as $\nu F_{\nu} / F_{b o l}$ in Figure 13. The spectra can also be used to compute the dimensionless ratios of $\nu F_{\nu} / F_{b o l}$ for each WISE band, which turn out to be well described by simple functions of the effective temperature $T_{e}$. The functional forms plotted in Figure 14 are given by:

$$
\frac{\nu F_{\nu}}{F_{b o l}}=0.16 \frac{15}{\pi^{4}} \frac{x^{4}}{e^{x}-1}
$$

with $x=h \nu /\left(1.34 k T_{e}\right)$ for band 1 in blue,

$$
\frac{\nu F_{\nu}}{F_{b o l}}=2.25 \min \left(T_{e} / 230,1\right)^{2} \frac{15}{\pi^{4}} \frac{x^{4}}{e^{x}-1}
$$

with $x=h \nu /\left(2.29 k T_{e}\right)$ for band 2 in green,

$$
\frac{\nu F_{\nu}}{F_{b o l}}=0.55 \frac{15}{\pi^{4}} \frac{x^{4}}{e^{x}-1}
$$

with $x=h \nu /\left(1.05 k T_{e}\right)$ for band 3 in orange, and

$\frac{\nu F_{\nu}}{F_{b o l}}=0.9 \begin{cases}\exp \left[-5.4 \log \left(T_{e} / 170\right)\right] & \text { if } T_{e}>317 \\ \exp \left[-20 \log \left(T_{e} / 170\right)^{2}\right] & \text { otherwise }\end{cases}$

for band 4 in red. Thus WISE band 2 sees a flux for $T_{\text {eff }}>230 \mathrm{~K}$ that is similar to a greybody with a temperature 2.25 times higher than $T_{\text {eff }}$, and with an emissivity 2.25 times higher than a blackbody. The fluxes in WISE bands 1 and 3 also look like higher temperature greybodies but with emissivities much smaller than a blackbody. The flux in WISE band 4 does not behave like a blackbody.

But when Eisenhardt et al. (2010) surveyed 10 square degrees with Spitzer to a flux limit at $4.5 \mu \mathrm{m} 20$ times deeper than the WISE all sky sensitivity, they found fewer very red sources than predicted by combining the Burrows et al. (2003) models with either the Chabrier (2003) log normal IMF or the Reid et al. (1999) power laws. The models could be brought into agreement with the observed counts by assuming that about $50 \%$ of the IRAC $4.5 \mu \mathrm{m}$ flux was suppressed by an absorber other than methane or water vapor. The amount of absorption needed is highly correlated with the assumed mass function, but the Eisenhardt et al. (2010) observation of about 10 brown dwarfs gives a modelindependent prediction that WISE should find about $10(41253 / 10) / 20^{1.5} \approx 400$ late-T and $\mathrm{Y}$ 
TABLE 2

NUMBER OF BROWN DWARFS WITH FLUXES GREATER THAN THE WISE $4.6 \mu$ M REQUIRED FLUX LIMIT OF $160 \mu \mathrm{Jy}$ FOR EITHER THE CHABRIER (2003) LOG NORMAL IMF (LGNM) OR REID ET AL. (1999) POWER LAWS WITH VARIOUS INDICES $\alpha$ FROM 0.4 TO 1.3 , WHILE SUPPRESSING A FRACTION $S$ OF THE $4.6 \mu \mathrm{M}$ FLUX.

\begin{tabular}{ccrrrr}
\hline \hline IMF & \multicolumn{1}{c}{$S$} & $T_{e}<300$ & $T_{e}<500$ & $T_{e}<750$ & $d<1.3 \mathrm{pc}$ \\
\hline $\operatorname{lgnm}$ & 0.000 & 7.1 & 228.5 & 1386.0 & 1.20 \\
$\cdots$ & 0.489 & 1.5 & 55.0 & 400.0 & 1.19 \\
& & & & & \\
0.4 & 0.000 & 2.6 & 79.0 & 520.8 & 0.47 \\
$\cdots$ & 0.125 & 1.9 & 58.6 & 400.0 & 0.47 \\
& & & & & \\
0.7 & 0.000 & 5.2 & 125.0 & 693.9 & 0.68 \\
$\cdots$ & 0.251 & 2.7 & 67.6 & 400.0 & 0.67 \\
& & & & & \\
1.0 & 0.000 & 10.7 & 203.2 & 952.9 & 1.06 \\
$\cdots$ & 0.375 & 3.8 & 76.9 & 400.0 & 1.03 \\
& & & & & \\
1.3 & 0.000 & 22.5 & 341.5 & 1356.1 & 1.82 \\
$\cdots$ & 0.493 & 5.3 & 88.9 & 400.0 & 1.72 \\
\hline \hline
\end{tabular}

class brown dwarfs. Table 2 shows the number of brown dwarfs in various temperature or distance bins that are detectable by WISE for different assumed mass functions. The age distribution is assumed to be uniform from $10^{8}$ to $10^{10}$ years, and the mass function is integrated from 1 to 80 $M_{J}$. For each mass and age bin the luminosity and effective temperature are found by interpolation. A fraction $S$ of the $4.6 \mu \mathrm{m}$ flux is then suppressed, following Golimowski et al. (2004), who found a suppression factor $1 /(1-S)=1.5$ to 2.5 , implying $S=0.5 \pm 0.1$. The volume in which the object could be detected is computed, multiplied times the space density for the mass and age bin, and summed over all masses and ages to give the numbers in Table 2. There are two lines for each mass function: the first does not include the W2 flux suppression, and the second has $S$ adjusted to give the expected 400 detectable objects with $T_{e}<750 \mathrm{~K}$. Matching the Eisenhardt et al. (2010) source density with a flux suppression in the range recommended by Golimowski et al. (2004) requires a high number density of brown dwarfs, as given by Chabrier (2003) or Reid et al. (1999) with a fairly steep power law index.

The WISE sample of brown dwarfs will be 3 magnitudes brighter than the Spitzer sample of Eisenhardt et al. (2010), and thus much easier to follow up with the HST or the Keck telescopes. Astrometric followup will be needed to pin down the properties of old, cold brown dwarfs.

\subsection{Ultra-Luminous Infrared Galaxies}

WISE will detect the most luminous galaxies in the Universe. The Ultra-Luminous Infrared Galaxies (ULIRGs) are due to mergers that lead to dust-enshrouded star formation and also to AGN activity as gas is disturbed out of stable circular orbits and falls into the central super-massive black holes in the merging galaxies. Figure 15 shows typical ULIRG and QSO spectra compared to the WISE flux limits.

In the concordance $\Lambda \mathrm{CDM}$ cosmology structure formation proceeds in a bottom-up manner, so large objects are formed by the mergers of smaller objects. The peak of large mergers occurs at redshifts 1 to 2 and leads to the peak in the star formation history of the Universe, and WISE has sufficient sensitivity at $22 \mu \mathrm{m}$ to see 


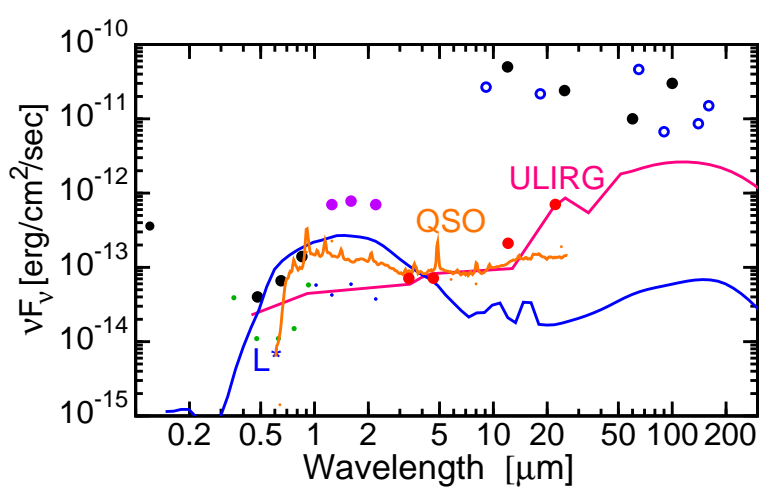

Fig. 15. - Plot showing the spectrum of an $L_{*}$ galaxy at redshift $z=0.33$, a ULIRG (FSC15307 redshifted to $z=3$ and scaled by a factor of 3 ), and observations of the $z=$ 6.42 QSO J1148+5251 with a scaled composite quasar spectrum in orange, compared to the WISE all-sky expected sensitivities in red. Other surveys are indicated with dot areas corresponding to their sky coverage: from the left, GALEX AIS Martin \& GALEX Science Team 2003), SDSS in green (York et al. 2000), DPOSS in black (Diorgovski et al. 1998), UKIDDS LAS in blue (Lawrence et al. 2007), 2MASS in magenta (Skrutskie et al. 2006), AKARI in blue open circles (Matsuhara et al. 2005), and the IRAS FSC in black (Moshir et al. 1992).

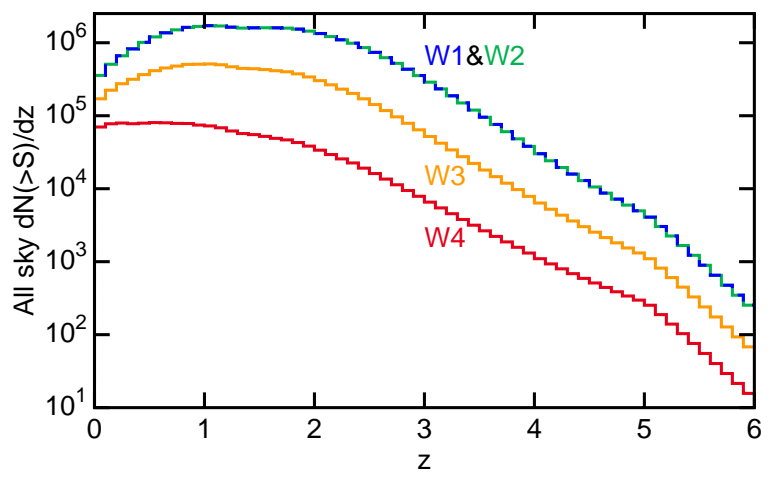

Fig. 16. - Number of QSOs and AGN brighter than the expected sensitivity in the 4 WISE bands over the entire sky, based on the Hopkins et al. (2007) luminosity function. The W1 and W2 curves lie almost on top of each other, with W3 and W4 giving lower counts. the top end of the luminosity function at redshift 3. As a result, WISE will detect many galaxies with $L>10^{13} L_{\odot}$. Quasar activity is also associated with mergers, and the evolving quasar luminosity function of Hopkins et al. (2007) predicts that WISE will see over $1.5 \times 10^{5}$ quasars at 22 $\mu \mathrm{m}$ and over 3.6 million quasars at $4.6 \mu \mathrm{m}$, with redshift distributions shown in Figure 16.

The abundance of ULIRGs as a function of luminosity and redshift will provide important information about the formation mechanisms that have led to the galaxies and clusters we see at low redshift today.

\subsubsection{Red/Dusty $A G N$ and $Q S O s$}

WISE will characterize and probe fully the extent of the population of obscured active galactic nuclei and will provide a highly uniform set of mid-infrared photometry for virtually all known Active Galactic Nuclei (AGN) and Quasi-Stellar Objects (QSOs). Most of what is known about the properties and evolution of AGN and QSOs has been deduced from studies of objects discovered in ultraviolet-excess and optical emission line surveys. However, infrared and radio surveys reveal that many AGN in the universe have remained hidden from short wavelength surveys because of reddening and obscuration by dust in and around their nuclei (Low et al. 1988; Webster et al. 1995). Estimates of the fraction of AGN missing from optical/UV selected samples vary considerably, ranging from 15 to over 50\% (Richardset al. 2003; Brown et al. 2007; Glikman et al. 2004, 2007). Such an obscured population may account for at least part of the hard X-ray background (Comastri et al. 1995), and it may contribute measurably to the far infrared background.

The most extensive searches for red AGN have been based on the 2MASS PSC, using either near-infrared color selection or combination with mid- and far-infrared and radio measurements (Cutri et al. 2001; Gregg et al. 2002; Glikman et al. 2007). These studies have found large numbers of predominantly low redshift AGN, adding considerably to the complete census of AGN in the local universe. However, 2MASS is a relatively shallow survey and is biased against the most heavily obscured AGN at higher redshifts. WISE will extend the 2MASS studies to 


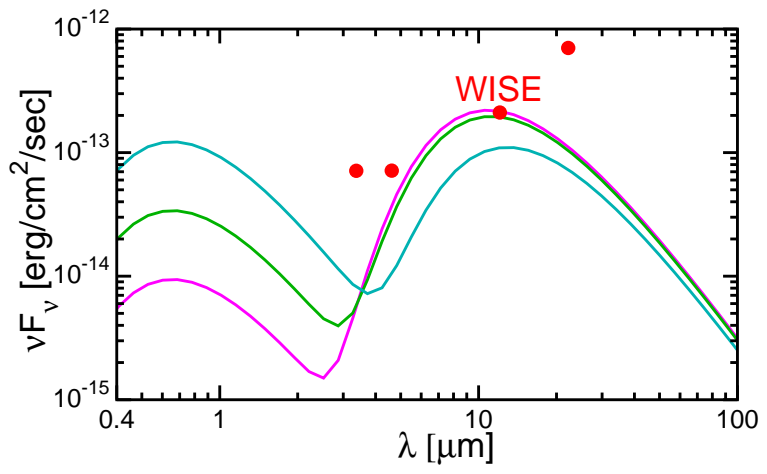

Fig. 17.- Fluxes from an $0.13 \mathrm{~km}$ diameter near Earth asteroid at a distance of $0.5 \mathrm{AU}$ with elongation $90^{\circ}$ for three albedos: $0.04,0.145$ and 0.52 from bottom to top on the left side of the plot. These models assume a Lambertian surface. The phase angle is $63^{\circ}$. The WISE $5 \sigma$ point source sensitivities for 8 stacked frames are shown as solid circles.

higher redshifts, probing the luminosity and number density evolution of red AGN over a significant fraction of the age of the universe.

One of the highest redshift QSOs, SDSS $1148+5251$, is shown in Figure 15, with data from Jiang et al. (2006) and a scaled composite spectrum constructed from Vanden Berk et al. (2001) and Glikman et al. (2006). A similar source is detectable by WISE at $3.4 \& 4.6 \mu \mathrm{m}$ over the whole sky, and also at $12 \mu \mathrm{m}$ with the better sensitivity achieved at the actual ecliptic latitude of this source $\left(\beta=46^{\circ}\right)$.

\subsection{Asteroids}

Asteroids are typically fairly dark, but do have a large range of albedos. As a result, the visual magnitude is an imprecise indicator of the size of an asteroid. Stuart \& Binzel (2004) show a range in albedos from 0.023 to 0.63 for near Earth asteroids, leading to a 5:1 range in estimated diameters and a 125:1 range in volumes. But the light that is not reflected is absorbed and reradiated in the thermal infrared. Figure 17 shows fluxes from an asteroid with low, median and high albedos. The ratio of optical to infrared fluxes gives the albedo, and the infrared flux and color temperature give a good indication of the diameter of an asteroid.

WISE is sensitive enough to see $1.2 \mathrm{~km}$ diam- eter asteroids in the main belt at 2.7 AU from the Sun, and $0.13 \mathrm{~km}$ diameter near Earth objects that are 0.5 AU from the Earth and 1.1 AU from the Sun. These limits are for objects with known orbits so that multiple frames can be stacked, and are for $5 \sigma$. This gives $10 \%$ accuracy in diameters, which is comparable to the errors caused by the unknown thermal inertia and rotation poles of most asteroids (Wright 2007). WISE will be able to provide radiometric diameters for about $3 \times 10^{5}$ asteroids using image stacking analysis. For an asteroid with a bolometric albedo of $A=5 \%$, the WISE stacked frame sensitivity of $800 \mu \mathrm{Jy}$ at 12 $\mu \mathrm{m}$ corresponds to an optical flux of roughly

$$
F_{\nu}(o p t) \approx \frac{\nu_{I R}}{\nu_{o p t}} \frac{A}{1-A} F_{\nu}(I R)=2 \mu \mathrm{Jy}
$$

which is an optical magnitude of 23. If good optical data are available, all of these asteroids will have well-determined albedos.

For new asteroids, a diameter of $2.4 \mathrm{~km}$ in the main belt or $0.25 \mathrm{~km}$ for a Near Earth Object (NEO) at a distance of $0.5 \mathrm{AU}$ gives enough signal-to-noise in a single frame to allow detections. The NEOWISE program (Mainzer et al. 2010) is searching the WISE data for moving objects and sending positional information to the IAU Minor Planet Center. WISE should detect more than $10^{5}$ asteroids on single frames.

WISE provides about 10 measurements of the flux spaced over 30 hours for a typical asteroid which can be used to derive rotation periods for tens of thousands of asteroids.

The accurate diameters for previously known asteroids plus new asteroids derived by the WISE task will allow a significantly more precise knowledge of the size frequency distribution (SFD) of sub-km NEOs. Since only a few dozen sub-km objects currently have diameters that have been accurately measured by either radar or thermal IR data, WISE data will significantly improve our knowledge of the SFD of these smaller objects. Diameters derived from WISE measurements will reduce uncertainties in the SFD associated with the traditional reliance on the optical $\mathrm{H}$ magnitude. Also, since infrared discoveries of new asteroids are relatively insensitive to albedo, the set of new discoveries made by WISE are much closer to a diameter-limited survey than optical surveys, resulting in much better SFDs. 
By observing asteroids at the time of their local afternoon and comparing this to their thermal fluxes in their local morning, WISE would enable measurement of the temperature differentials that give rise to the Yarkovsky effect, a phenomenon that is often the dominant uncertainty in the long term orbit propagation for small asteroids. Measuring the Yarkovsky effect via the thermal infrared provides rather different information for a different population than the radar method (Chesley et al. 2003). The radar technique uses precision observations of an asteroid's trajectory over a decade to look for small changes to the ephemeris caused by the Yarkovsky force. In order to obtain sufficient astrometric precision, only NEOs which are very nearby can be observed. Radar data allow one to compute the tangential acceleration $a$ produced by the Yarkovsky effect. However, using infrared to look for temperature differentials between an asteroid's local morning and evening requires only two epochs of observation separated by 3-8 months. The infrared technique is not limited to NEOs and will also work for Main Belt asteroids such as the Baptistina family. The infrared data allow one to compute the diameter which gives the mass $m$, and the morning $v s$. evening difference gives the tangential force $F$. Thus combining IR and radar data allows measurements of all the quantities in $F=m a$. For asteroids in a family, the change in semi-major axis divided by the age of the family gives an estimate for the acceleration $a$, so the IR data can be used to verify the time since the collision that created the family. The hypothesis (Bottke et al. 2007) that the K/T impactor was part of the Baptistina family depends on the estimated $160 \mathrm{Myr}$ age of this family, so measuring the Yarkovsky force and diameter for a sample of Baptistina family members will provide a valuable test of this idea. The infrared Yarkovsky detection technique is improved by knowledge of an objects lightcurve, well measured by WISE.

\subsection{Other Science Goals}

An all-sky survey in the thermal infrared can be used to address many scientific questions, not all of which require surveying the whole sky. But by surveying the whole sky, WISE will enable these projects among many others:

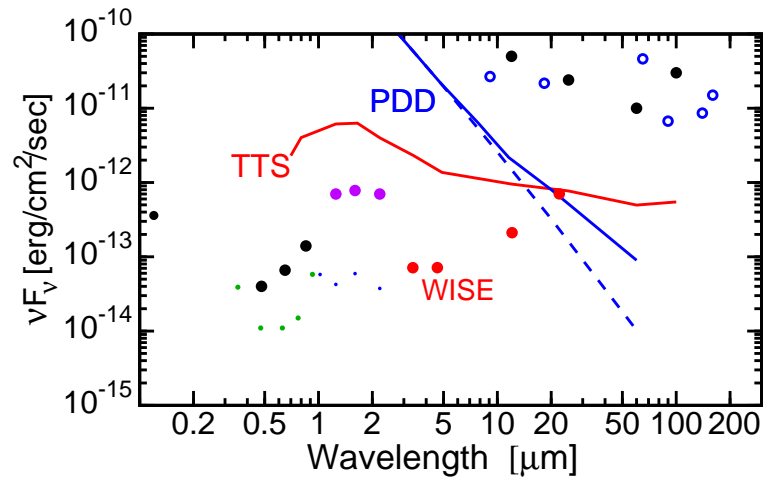

Fig. 18. - Figure showing an M0 T Tauri star at a distance of $2.5 \mathrm{kpc}$ (TTS) and an A2 star with a planetary debris disk at $300 \mathrm{pc}$ (PDD) compared to the WISE expected sensitivities. The unlabeled points for other surveys are the same as in Figure 15.

\subsubsection{Comet Trails and Zodiacal Bands}

WISE will study the nearest planetary debris disk: our own zodiacal cloud and asteroid system. The interplanetary dust cloud is a complex distribution of dust grains from numerous sources evolving under the influence of planetary perturbations, collisional evolution and solar radiation sources. The zodiacal dust cloud was long thought to come from comets (Whipple 1955, 1967, 1976) but current estimates of cometary dust production are an order of magnitude below that needed to maintain the cloud. The IRAS dust bands show that asteroids are a substantial contributor to the interplanetary dust cloud. There are at least seven dust band pairs girdling the ecliptic. Comet debris trails were also discovered by IRAS (Sykes et al. 1986, 1990) which provided more than 100 observations of the trails associated with 8 short period comets. WISE scans through the ecliptic 30 times per day which will permit frequent observations of dust bands and comet trails with an angular resolution many times better than IRAS. This allows a detailed assessment of the population and evolution of these structures in the zodiacal cloud.

\subsubsection{Young Stars and Debris Disks}

WISE will provide a robust statistical database for studying star formation, the evolution of circumstellar disks, and the dissipation of zodiacal clouds around stars of all masses, extending even 
into the brown dwarf mass regime. Detecting the small IR excess from the later stages of disk dissipation requires the ability to accurately predict the long wavelength photospheric emission and the ability to accurately measure the total long wavelength emission. The simultaneous 4 color photometry provided by WISE will enable the required predictions, and the sensitivity of WISE will enable detection of optically thick disk emission for solar type stars out to $1 \mathrm{kpc}$ distance and down to the hydrogen burning limit in the Taurus and Ophiucus star forming regions. Because young stars are variable, the simultaneous fourcolor coverage of WISE provides a precise means of characterizing thermal excess from circumstellar disks or zodiacal material. (A possible second coverage of the object by WISE 6 months after the first will even provide clues as to the nature of the variability, e.g. extinction vs. accretion.)

Because WISE is an all-sky survey it will reveal isolated young stars well outside obvious starforming regions. For example, two of the nearest clusters (of any age) were discovered only within the last decade though their YSO-like properties: the TW Hydrae Association $(\mathrm{d}=55 \mathrm{pc}$, by infrared emission) and the Eta Chamaeleonis Cluster $(\mathrm{d}=96 \mathrm{pc}$, by X-ray emission). Both have about a dozen known members and are younger than 10 Myr. Surprisingly neither is associated with a molecular cloud. TW Hya covers more than 700 sq. degrees. A complete survey would be an impossible task for missions like Spitzer but is the natural focus of WISE. Such intermediate-age very-nearby clusters provide an ideal laboratory for studying the late stages of planetary accretion.

WISE will find young stars which are still accreting molecular cloud material over a substantial fraction of the Galaxy: WISE will measure stars with optically-thick circumstellar disk emission down to the hydrogen-burning limit $(0.08$ $\mathrm{M}_{\odot}$ ) out to 500 parsecs (e.g. the Orion molecular cloud). Solar-type "Class I" and T-Tauri stars with optically-thick disks will be found to substantial distances (Figure 18 shows the M0 T Tauri star Sz 82 scaled to a distance of $2.5 \mathrm{kpc}$ ), thanks to their strong excesses at 12 and $22 \mu \mathrm{m}$. Expanding upon Spitzer's survey of selected regions of a few molecular clouds, WISE will provide a full inventory of low-luminosity young stellar objects in many dozens of star forming clouds extending over the entire sky. WISE will also discover hundreds of the very youngest stars: the elusive "Class 0 " sources. These objects, which are in the early stages of gravitational collapse, are rare because they remain in this phase for less than 100,000 yr. They are deeply embedded in dust, so they are visible only at wavelengths longer than $20 \mu \mathrm{m}$, although they may be seen in scattered light in the mid-IR.

WISE will survey planetary debris disks around thousands of stars in the solar neighborhood. During the early stages of planet formation micronsized grains accrete into successively larger particles. The resulting reduction in grain surface area makes the circumstellar disk optically thin, and the infrared excess substantially decreases. The disk does not clear uniformly due to both the variation in accretion/dynamical timescale with distance from the star, and the shepherding effects of forming planets (Kuchner \& Holman 2003). This non-uniformity is evident in the distribution of infrared excess as a function of wavelength through the mid-infrared. WISE will exploit these signatures to reveal the systematics of and refine the timescales for disk clearing and solar system formation.

Although the planetesimal/planet formation phase is short-lived, dust excess signatures can persist into the main-sequence phase as demonstrated in stars like Vega by IRAS (Aumann et al. 1984). These excesses arise from small grains with Poynting-Robertson lifetimes of tens of thousands of years. Their existence argues that they must be actively replenished by the erosion of planetesimals and thus they are signatures of successful planetary formation. Indeed, high resolution 20 $\mu \mathrm{m}$ imaging of infrared-excess objects like HR4796 reveal dust rings which may betray the existence of invisible planets. Figure 18 also shows the known debris disk system $\zeta$ Lep scaled to a distance of $300 \mathrm{pc}$. With the short wavelength WISE bands pinning down the photospheric flux, the excess at $22 \mu \mathrm{m}$ is easily detectable.

The sensitivity to see the photospheric flux and enough wavelength coverage to predict the photometric flux are, once again, the key to revealing excesses from eroding planetesimals. In addition to studying the statistical distribution of such small IR excesses, of great interest to the TPF mission, WISE will provide JWST with hundreds 
of targets for high-resolution imaging akin to the ground-based and HST images of HR4796.

\subsubsection{Interstellar Dust}

WISE detects most components of interstellar dust and produces very good maps of interstellar dust in the Galaxy. The 3.4 and $12 \mu \mathrm{m}$ filters include prominent $\mathrm{PAH}$ emission features, the $4.6 \mu \mathrm{m}$ filter measures the continuum emission from very small grains, and the $22 \mu \mathrm{m}$ filter sees both stochastic emission from small grains and the Wien tail of thermal emission from large grains. The WISE survey will map the high latitude dust to a sensitivity 60 times better than IRAS at 22 $\mu \mathrm{m}$ and 200 times better at $12 \mu \mathrm{m}$. For typical high latitude dust showing PAH emission the 3.4 $\mu \mathrm{m}$ map will provide a signal to noise ratio comparable in sensitivity to the $12 \mu \mathrm{m}$ map. The WISE survey thus extends the mapping of high latitude dust to cover the entire sky, filling in the holes left by IRAS in the dimmest regions of sky, and extending the Spitzer legacy projects GLIMPSE and MIPSGAL to the entire sky. The $3.4 \mu \mathrm{m}$ map will provide the first high sensitivity map of extended emission of the entire sky at this wavelength.

These extended emission maps will allow study of the composition and detailed structure of interstellar dust. Unique to WISE, a comparison of the $3.3 \mu \mathrm{m}$ PAH feature with other PAH emission features in diffuse clouds can yield information on $\mathrm{PAH}$ composition. Results of the study of dust in the Galaxy can also be compared to dust emission detected by WISE in nearby galaxies.

\subsubsection{Galactic Structure}

Roughly half of the sky is dominated by the Milky Way's 400 billion stars, many of which are variable in the infrared. With deep simultaneous near- and mid-infrared observations, unique in an all-sky survey, the 4-color WISE will address global issues of Galactic stellar structure and populations: the disk's warp and flare; the nature of the bar, Gould's Belt, ring, and bulge; a detailed census of evolved stars; and the pursuit of halo star-streams from globular clusters and unmerged Galactic dwarf satellites. WISE complements Spitzer's Legacy Project, GLIMPSE, which surveyed $<300$ square degrees of the inner plane mostly constrained to $|b|<1^{\circ}$. WISE will cover all the Galactic plane, and secure critical off-plane starcounts to probe the vertical population structure in the arms and spurs, and scale heights of the central bar, molecular ring and bulge (disk contamination diminishes rapidly off the plane). Although the stellar counts are confusion limited near the Galactic plane with the WISE beam size and high sensitivity, the color-magnitude diagrams are robust to confusion effects; and thus delineation of differing stellar populations is still viable in the Galactic plane.

Off plane, unconstrained by confusion, WISE offers unparalleled infrared survey depth, uniquely capable of all-sky detection of even normal halo stars. The "Spaghetti Survey" (Morrison et al. 2000) of the halo uses optical photometry and spectroscopy to identify distant G-/K-giants and kinematic substructure caused by the destruction of accreted satellites. WISE will aid the optical quest for extra-tidal K-giants, detecting such stars to $70 \mathrm{kpc}$ at $3.4 \mu \mathrm{m}$ and $45 \mathrm{kpc}$ at $4.6 \mu \mathrm{m}$, far beyond the depth of 2MASS at $2.15 \mu \mathrm{m}$. Comparing the optical spectra and WISE data on the quasi-continuum $(3.4 \mu \mathrm{m})$ and $\mathrm{CO}$ fundamental $(4.6 \mu \mathrm{m})$ in these cool stars to synthetic spectra will enable the recognition of those stars that belong to remnant stellar swarms from partly digested satellites.

\subsubsection{Nearby Galaxies}

The angular resolution and sensitivity of WISE allow detailed study of the internal structure of galaxies in the local Universe, whose properties provide our basic understanding of high-redshift galaxies. For the $\sim 5000$ galaxies within $20 \mathrm{Mpc}$ of the Sun, WISE spatial resolution is $<500$ par$\operatorname{secs}(<1 \mathrm{kpc}$ at $22 \mu \mathrm{m})$. Of this set, nearly 400 will be highly resolved, delineating globular clusters, giant molecular clouds and other discrete sites of star formation. Fig [19] shows WISE images of 4 well resolved nearby galaxies. The short mid-IR bands of WISE are an effective probe of the underlying stellar population, and are nearly immune to extinction. The long bands are sensitive to hot dust associated with star formation, thus bridging the time span between evolving generations of stars. WISE data will complement the Spitzer Infrared Nearby Galaxies Survey Legacy project (SINGS, Kennicutt et al. 2004), expanding the SINGS sample of 75 galaxies to many 

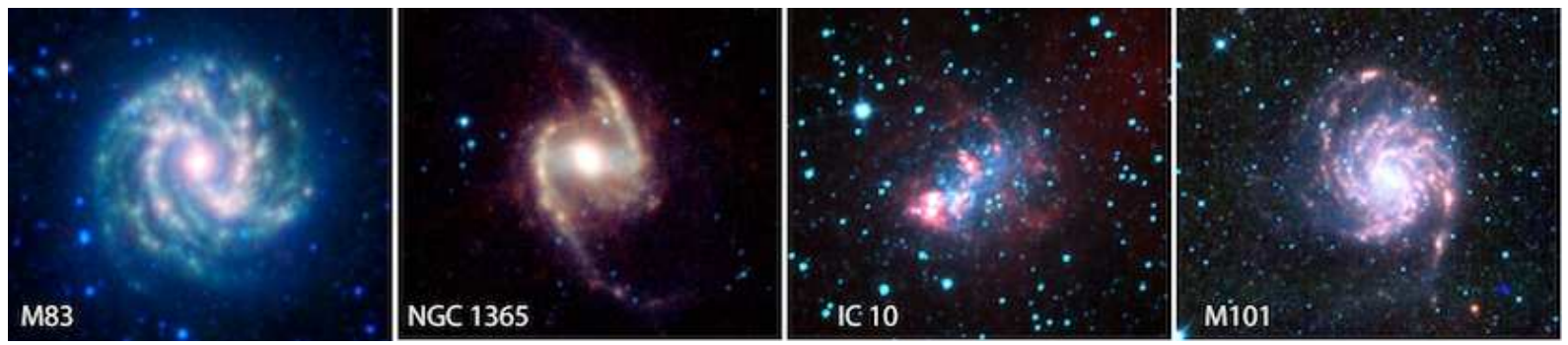

Fig. 19.- Four nearby spiral and irregular galaxies that are well resolved by WISE.

thousands of galaxies, covering the widest range in morphological type. It will also supplement the IRAC 3.6/4.5 $\mu \mathrm{m}$ Spitzer Survey of Stellar Structure in Galaxies (S4G, Sheth et al. 2008): WISE will provide the crucial mid-IR tracers of star formation activity for this complete sample of galaxies within $30 \mathrm{Mpc}$.

\subsubsection{Galaxy Clusters}

WISE will provide a census of star formation in very large samples of normal galaxies and clusters out to cosmological distances. WISE will detect over 100 million galaxies in at least one band, and many million galaxies in all four of its bands. The short wavelength W1-W2 color will be a useful redshift indicator for galaxies out to $z=0.5$, and the flux measurements will provide an estimate for the total stellar mass of galaxies at $z<0.5$ by measuring the rest frame 1.6-2.2 $\mu \mathrm{m}$ emission (Gavazzi et al. 1996). WISE will thus provide a global history of star formation in massive field and cluster galaxies over the past 5 billion years.

Cluster galaxies generally contain stellar populations that are old relative to those in the surrounding field galaxies, so the contrast of galaxy clusters relative to the field increases with redshift when viewed in the IR. By virtue of covering the entire sky, WISE will be able to detect all massive $\left(M>10^{14} M_{\odot}\right)$ galaxy clusters up to $z \sim 0.5$, enabling the most complete census of the stellar mass in dense environments when used in conjunction with large-area optical imaging surveys such as SDSS, PanStarrs, DES, and LSST. When used with the higher spatial resolution optical imaging from these surveys, which can help to overcome the confusion limit in the WISE images, WISE will also be able to identify even higher redshift clusters $(0.5<z<1)$ over thousands of square degree areas at the poles where the larger than average number of WISE scans will lower the noise in the W1 data.

\section{Data Products}

WISE will produce an image atlas in its 4 colors that will be a stack of all the multiple frames covering each part of the sky. A catalog of sources extracted from these images will also be produced. These data products are modeled on the 2MASS image atlas and the 2MASS point source catalog. WISE is not planning to produce a separate extended source catalog.

In addition, the NEOWISE project is supporting the release of the individual WISE frames and a database of all the sources extracted from the individual frames. This dataset will be very valuable for retrospective studies of asteroids discovered in the future.

There are two data releases scheduled for WISE. The first release will be a preliminary catalog and image atlas based on early data and covering more than $55 \%$ of the sky, to be released in April 2011. The preliminary catalog will be complete to a depth of $20 \sigma$. A final data release covering the entire sky to a depth of $5 \sigma$ will be released 17 months after the last data are taken.

\section{Prospects for Followup Observations}

The low background from space makes the WISE survey very sensitive, and allows it to cover the largest possible solid angle while still reaching fairly low flux levels. The faintness of the sources found by WISE makes followup with ground-based telescopes quite difficult in the thermal infrared. However, the time required for a detection scales like $B / D^{4}$ for background $B$ and telescope diam- 
eter $D$, assuming diffraction-limited optics, so it will be possible to confirm WISE sources with ground-based 10 meter class telescopes, since the $(10 / 0.4)^{4}=390,625$ diameter factor almost compensates for the factor of $2.5 \times 10^{6}$ in the backgrounds. Since WISE only integrates for $1.2 \mathrm{~min}-$ utes on a typical source, 15 minutes on a 10 meter class telescope can surpass the WISE sensitivity on point sources and give much better angular resolution. Even though ground-based followup in the thermal infrared is possible, the most useful ground-based followups will probably be at complementary wavelengths. Examples include ground-based $J$ band measurements of WISE brown dwarfs, ground-based optical astrometry of WISE asteroids, ground-based optical spectroscopy of WISE ULIRGs and AGN, and photometric redshifts of galaxies based on optical and infrared colors.

The James Webb Space Telescope will also experience the low background intensity seen by WISE, will cover the WISE passbands, and will be much larger than WISE. Thus JWST will be the most capable platform for studying WISE sources in the thermal infrared, since it will have both a large diameter and low background. WISE data at 3.4 and $4.6 \mu \mathrm{m}$ will be used to improve the JWST guide star catalog by providing improved count rate predictions and more recent astrometry.

\section{Conclusion}

WISE has exploited the low infrared background in space and the power of large format infrared arrays to map the entire sky with unprecedented sensitivity and angular resolution between 3.4 and $22 \mu \mathrm{m}$. The WISE catalog and image atlas will be extremely valuable for a large number of astrophysical investigations ranging from asteroid albedos in the solar system through the stellar population of the solar neighborhood out to the most luminous star forming galaxies in the visible Universe.

This publication makes use of data products from the Wide-field Infrared Survey Explorer, which is a joint project of the University of California, Los Angeles, and the Jet Propulsion Laboratory/California Institute of Technology, funded by the National Aeronautics and Space Adminis- tration.

\section{REFERENCES}

Aumann, H. H., Beichman, C. A., Gillett, F. C., de Jong, T., Houck, J. R., Low, F. J., Neugebauer, G., Walker, R. G., \& Wesselius, P. R. 1984, ApJ, 278, L23

Beichman, C. A., Neugebauer, G., Habing, H. J., Clegg, P. E., \& Chester, T. J., eds. 1988, Infrared astronomical satellite (IRAS) catalogs and atlases. Volume 1: Explanatory supplement, Vol. 1

Bottke, W. F., Vokrouhlický, D., \& Nesvorný, D. 2007, Nature, 449, 48

Brown, M. J. I. et al. 2006, ApJ, 638, 88

Burrows, A., Sudarsky, D., \& Lunine, J. I. 2003, ApJ, 596, 587

Chabrier, G. 2002, ApJ, 567, 304

—. 2003, PASP, 115, 763

Chesley, S. R., Ostro, S. J., Vokrouhlický, D., Čapek, D., Giorgini, J. D., Nolan, M. C., Margot, J.-L., Hine, A. A., Benner, L. A. M., \& Chamberlin, A. B. 2003, Science, 302, 1739

Cohen, M. 2009, AJ, 137, 3449

Cohen, M., Walker, R. G., Barlow, M. J., \& Deacon, J. R. 1992, AJ, 104, 1650

Comastri, A., Setti, G., Zamorani, G., \& Hasinger, G. 1995, A\&A, 296, 1

Cutri, R. M., Nelson, B. O., Kirkpatrick, J. D., Huchra, J. P., \& Smith, P. S. 2001, in Bulletin of the American Astronomical Society, Vol. 33, Bulletin of the American Astronomical Society, 829

Djorgovski, S. G., Gal, R. R., Odewahn, S. C., de Carvalho, R. R., Brunner, R., Longo, G., \& Scaramella, R. 1998, in Wide Field Surveys in Cosmology, ed. S. Colombi, Y. Mellier, \& B. Raban, 89

Duval, V. G., Irace, W. R., Mainzer, A. K., \& Wright, E. L. 2004, in Presented at the 
Society of Photo-Optical Instrumentation Engineers (SPIE) Conference, Vol. 5487, Optical, Infrared, and Millimeter Space Telescopes. Edited by Mather, John C. Proceedings of the SPIE, Volume 5487, pp. 101-111 (2004)., ed. J. C. Mather, 101-111

Eisenhardt, P. R. M. et al. 2010, AJ, 139, 2455

Gavazzi, G., Pierini, D., \& Boselli, A. 1996, A\&A, 312,397

Glikman, E., Gregg, M.D., Lacy, M., Helfand, D.J., Becker, R.H. \& White, R.L. 2004, ApJ, 607,60

Glikman, E., Helfand, D. J., \& White, R. L. 2006, ApJ, 640, 579

Glikman, E., Helfand, D.J., White, R.L. \& Becker, R.H. 2007, ApJ, 667, 673

Golimowski, D. et al. 2004, AJ, 127, 3516

Gregg, M.D, Lacy, M., White, R.L., Glikman, E., Helfand, D., Becker, R.H. \& Brotherton, M.S. 2002, ApJ, 564, 133

Heinrichsen, I. \& Wright, E. L. 2006, in Presented at the Society of Photo-Optical Instrumentation Engineers (SPIE) Conference, Vol. 6270, Observatory Operations: Strategies, Processes, and Systems. Edited by Silva, David R.; Doxsey, Rodger E.. Proceedings of the SPIE, Volume 6270, pp. 62701C (2006).

Hopkins, P. F., Richards, G. T., \& Hernquist, L. 2007, ApJ, 654, 731

Ishihara, D. et al. 2010, A\&A, 514, A1

Jiang, L. et al. 2006, AJ, 132, 2127

Kennicutt, R., Armus, L., Calzetti, D., Dale, D., Draine, B., Engelbracht, C., Gordon, K., Helou, G., Hollenbach, D., Leitherer, C., Malhotra, S., Regan, M., Rieke, G., Rieke, M., \& Thornley, M. 2004, in Spitzer Proposal ID \#159

Kirkpatrick, J. D. 2005, ARA\&A, 43, 195

Kuchner, M. J. \& Holman, M. J. 2003, ApJ, 588, 1110
Kurucz, R. 1993, ATLAS9 Stellar Atmosphere Programs and $2 \mathrm{~km} / \mathrm{s}$ grid. Kurucz CD-ROM No. 13. Cambridge, Mass.: Smithsonian Astrophysical Observatory, 1993., 13

Larsen, M. F. \& Schick, S. 2005, in Presented at the Society of Photo-Optical Instrumentation Engineers (SPIE) Conference, Vol. 5904, Cryogenic Optical Systems and Instruments XI. Edited by Heaney, James B.; Burriesci, Lawrence G. Proceedings of the SPIE, Volume 5904, pp. 166-177 (2005)., ed. J. B. Heaney \& L. G. Burriesci, 166-177

Lawrence, A., Warren, S. J., Almaini, O., Edge, A. C., Hambly, N. C., Jameson, R. F., Lucas, P., Casali, M., Adamson, A., Dye, S., Emerson, J. P., Foucaud, S., Hewett, P., Hirst, P., Hodgkin, S. T., Irwin, M. J., Lodieu, N., McMahon, R. G., Simpson, C., Smail, I., Mortlock, D., \& Folger, M. 2007, MNRAS, 379, 1599

Liu, F., Cutri, R., Greanias, G., Duval, V., Eisenhardt, P., Elwell, J., Heinrichsen, I., Howard, J., Irace, W., Mainzer, A., Razzaghi, A., Royer, D., \& Wright, E. L. 2008, in Presented at the Society of Photo-Optical Instrumentation Engineers (SPIE) Conference, Vol. 7017, Society of Photo-Optical Instrumentation Engineers (SPIE) Conference Series, pp. 70170M70170M-12

Low, F. J., Cutri, R. M., Huchra, J. P., \& Kleinmann, S. G. 1988, ApJ, 327, L41

Mainzer, A. K., Eisenhardt, P., Wright, E. L., Liu, F.-C., Irace, W., Heinrichsen, I., Cutri, R., \& Duval, V. 2005, in Presented at the Society of Photo-Optical Instrumentation Engineers (SPIE) Conference, Vol. 5899, UV/Optical/IR Space Telescopes: Innovative Technologies and Concepts II. Edited by MacEwen, Howard A. Proceedings of the SPIE, Volume 5899, pp. 262273 (2005)., ed. H. A. MacEwen, 262-273

Mainzer, A. et al. 2010, in preparation.

Martin, C. \& GALEX Science Team. 2003, in Bulletin of the American Astronomical Society, Vol. 35, Bulletin of the American Astronomical Society, 1363

Masci, F. J. \& Fowler, J. W. 2009, in Astronomical Society of the Pacific Conference Series, Vol. 
411, Astronomical Society of the Pacific Conference Series, ed. D. A. Bohlender, D. Durand, \& P. Dowler, 67

Matsuhara, H., Shibai, H., Onaka, T., \& Usui, F. 2005, Advances in Space Research, 36, 1091

Méndez, B. J. H. 2008, in Astronomical Society of the Pacific Conference Series, Vol. 389, EPO and a Changing World: Creating Linkages and Expanding Partnerships, ed. C. Garmany \& M. G. Gibbs Moody, J. W., 385

Morrison, H. L., Mateo, M., Olszewski, E. W., Harding, P., Dohm-Palmer, R. C., Freeman, K. C., Norris, J. E., \& Morita, M. 2000, AJ, 119,2254

Moshir, M., Kopman, G., \& Conrow, T. A. O. 1992, IRAS Faint Source Survey, Explanatory supplement version 2 (Pasadena: Infrared Processing and Analysis Center, California Institute of Technology, 1992, edited by Moshir, M.; Kopman, G.; Conrow, T.)

Murakami, H. et al. 2007, PASJ, 59, 369

Naes, L., Lloyd, B., \& Schick, S. 2008, in American Institute of Physics Conference Series, Vol. 985, Advances in Cryogenic Engineering, ed. J. G. Weisend, J. Barclay, S. Breon, J. Demko, M. Dipirro, J. P. Kelley, P. Kittel, A. Klebaner, A. Zeller, M. Zagarola, S. van Sciver, A. Rowe, J. Pfotenhauer, T. Peterson, \& J. Lock, 815822

Neugebauer, G. et al. 1984, ApJ, 278, L1

Price, S. D., Paxson, C., Engelke, C., \& Murdock, T. L. 2004, AJ, 128, 889

Reid, I. N., Kirkpatrick, J. D., Liebert, J., Burrows, A., Gizis, J. E., Burgasser, A., Dahn, C. C., Monet, D., Cutri, R., Beichman, C. A., \& Skrutskie, M. 1999, ApJ, 521, 613

Richards, G. T. et al. 2003, AJ, 126, 1131

Schick, S. \& Lloyd, B. 2009, in Presented at the Society of Photo-Optical Instrumentation Engineers (SPIE) Conference, Vol. 7439, Society of Photo-Optical Instrumentation Engineers (SPIE) Conference Series, pp. 743917743917-7
Schneps, M. H. \& Wright, N. 1980, S\&T, 59, 195

Schwalm, M., Barry, M., Perron, G., Sampath, D., LaMalva, F., Guregian, J., \& Crowther, B. 2005, in Presented at the Society of Photo-Optical Instrumentation Engineers (SPIE) Conference, Vol. 5904, Cryogenic Optical Systems and Instruments XI. Edited by Heaney, James B.; Burriesci, Lawrence G. Proceedings of the SPIE, Volume 5904, pp. 178-184 (2005)., ed. J. B. Heaney \& L. G. Burriesci, 178-184

Sheth, K. et al. 2008, in Spitzer Proposal ID \#60007

Skrutskie, M. F. et al. 2006, AJ, 131, 1163

Smith, B. J., Price, S. D., \& Baker, R. I. 2004, ApJS, 154, 673

Stuart, J. S. \& Binzel, R. P. 2004, Icarus, 170, 295

Sykes, M. V., Lebofsky, L. A., Hunten, D. M., \& Low, F. 1986, Science, 232, 1115

Sykes, M. V., Lien, D. J., \& Walker, R. G. 1990, Icarus, 86, 236

Szalay, A. S., Connolly, A. J., \& Szokoly, G. P. 1999, AJ, 117, 68

Tokunaga, A. T. \& Vacca, W. D. 2005, PASP, 117, 1459

Vanden Berk, D. E. et al.2001, AJ, 122, 549

Webster, R. L., Francis, P. J., Peterson, B. A., Drinkwater, M. J., \& Masci, F. J. 1995, Nature, 375,469

Whipple, F. L. 1955, ApJ, 121, 750

—. 1967, SAO Special Report, 239, 1

Whipple, F. L. 1976, in Lecture Notes in Physics, Berlin Springer Verlag, Vol. 48, Interplanetary Dust and Zodiacal Light, ed. H. Elsaesser \& H. Fechtig, 403-415

Wright, E. L. 1985, PASP, 97, 451

Wright, E. L. 2007, in AAS/Division for Planetary Sciences Meeting Abstracts, Vol. 39, AAS/Division for Planetary Sciences Meeting Abstracts, 483 
York, D. G. et al. 2000, AJ, 120, 1579

Zacharias, N. et al. 2010, AJ, 139, 2184

This 2-column preprint was prepared with the AAS IATEX macros v5.2. 